\title{
Toeplitz Operators Acting on True-Poly-Bergman Type Spaces of the Two-Dimensional Siegel Domain: Nilpotent Symbols
}

\author{
Yessica Hernández-Eliseo, Josué Ramírez-Ortega $\mathbb{D}$, and Francisco G. Hernández-Zamora
}

Universidad Veracruzana, Mexico

Correspondence should be addressed to Josué Ramírez-Ortega; josramirez@uv.mx

Received 10 August 2020; Revised 24 October 2020; Accepted 10 November 2020; Published 13 January 2021

Academic Editor: Aurelian Gheondea

Copyright (c) 2021 Yessica Hernández-Eliseo et al. This is an open access article distributed under the Creative Commons Attribution License, which permits unrestricted use, distribution, and reproduction in any medium, provided the original work is properly cited.

We describe certain $C^{*}$-algebras generated by Toeplitz operators with nilpotent symbols and acting on a poly-Bergman type space of the Siegel domain $D_{2} \subset \mathbb{C}^{2}$. Bounded measurable functions of the form $c\left(\operatorname{Im} \zeta_{1}, \operatorname{Im} \zeta_{2}-\left|\zeta_{1}\right|^{2}\right)$ are called nilpotent symbols. In this work, we consider symbols of the form $a\left(\operatorname{Im} \zeta_{1}\right) b\left(\operatorname{Im} \zeta_{2}-\left|\zeta_{1}\right|^{2}\right)$, where both limits $\lim _{s \rightarrow 0^{+}} b(s)$ and $\lim _{s \rightarrow+\infty} b(s)$ exist, and $a(s)$ belongs to the set of piecewise continuous functions on $\overline{\mathbb{R}}=[-\infty,+\infty]$ and having one-side limit values at each point of a finite set $S \subset \mathbb{R}$. We prove that the $C^{*}$-algebra generated by all Toeplitz operators $T_{a b}$ is isomorphic to $C(\bar{\Pi})$, where $\bar{\Pi}=\overline{\mathbb{R}} \times \overline{\mathbb{R}}_{+}$and $\overline{\mathbb{R}}_{+}=[0,+\infty]$.

\section{Introduction}

In the study of Toeplitz operators, one of the common strategies consists in selecting a set of symbols $E \subset L^{\infty}$ in such a way that the algebra generated by Toeplitz operators with symbols in $E$ can be described up to isomorphism, say, with an algebra of continuous functions or finding its spectrum. In this paper, we study Toeplitz operators with nilpotent symbols and acting on a poly-Bergman type space of the Siegel domain $D_{2} \subset \mathbb{C}^{2}$. In [1-3], the authors have fully described all commutative $C^{*}$-algebras generated by Toeplitz operators with symbols invariant under the action of a maximal abelian subgroup of biholomorphisms and acting on the Bergman spaces of both the unit disk $\mathbb{D}$ and the Siegel domain $D_{n} \subset \mathbb{C}^{n}$. For the unit disk, they discovered three families of symbols associated to commutative $C^{*}$-algebras of Toeplitz operators, while for the Siegel domain, they found $n+2$ classes of symbols. Each class of symbols is invariant under the action of a maximal abelian group of biholomorphism. Certainly, one can use these classes of symbols to study Toeplitz operators acting on poly-Bergman type spaces of the unit disk or the Siegel domain.

Let $\Pi=\{z=x+i y \in \mathbb{C}: y>0\}$ be the upper half-plane. Toeplitz operators with vertical symbols, which depend on
$y=\operatorname{Im} z$, and acting on Bergman type spaces have been studied. In [4-9], the authors proved that the algebra generated by Toeplitz operators with vertical symbols and acting on the weighted Bergman space $\mathscr{A}_{\lambda}^{2}(\Pi)$ is isometrically isomorphic to the algebra $\operatorname{VSO}\left(\mathbb{R}_{+}\right)$of all bounded functions that are very slowly oscillating on $\mathbb{R}_{+}$. Taking vertical symbols having limit values at $y=0$ and $y=\infty$, in $[10,11]$, the authors found that $\overline{\mathbb{R}}_{+}=[0,+\infty]$ is the spectrum of the algebra generated by all Toeplitz operators on the true-poly Bergman space $\mathscr{A}_{(n)}^{2}(\Pi)$. Similar research was made for Toeplitz operators on poly-Bergman spaces with homogeneous symbols $([12,13])$. Other works about it were made in [14-16], where the authors studied Toeplitz operators acting on $\mathscr{A}_{(n)}^{2}(\Pi)$ from the point of view of wavelet spaces. On the other hand, in $[17,18]$, the authors studied Toeplitz operators on the Fock space $F_{1}^{2}(\mathbb{C})$ with radial and bounded horizontal symbols; they found that spectral functions are uniformly continuous with respect to an adequate metric. Taking horizontal symbols having one-side limits at $x= \pm \infty$, in $[19,20]$, the authors studied Toeplitz operators acting on poly-Fock spaces $F_{k}^{2}(\mathbb{C})$; they found the spectrum of the $C^{*}$-algebra generated by such Toeplitz operators. Even though the authors described the $C^{*}$-algebras generated by all spectral 
functions, the spectrum of the algebras is not fully understood in some cases; for this reason, additional conditions on the symbols are imposed.

In $[1,2]$, the authors made remarkable research on the study of Toeplitz operators acting on the Bergman space of the Siegel domain $D_{n} \subset \mathbb{C}^{n}$. In particular, they studied the $C^{*}$-algebra $T_{N_{n}}$ generated by all Toeplitz operators with bounded nilpotent symbols, which are functions of the form $a(\zeta)=a\left(\operatorname{Im} \zeta_{1}, \cdots, \operatorname{Im} \zeta_{n-1}, \operatorname{Im} \zeta_{n}-\left|\zeta^{\prime}\right|^{2}\right)$, where $\zeta^{\prime}=\left(\zeta_{1}, \cdots\right.$ ,$\left.\zeta_{n-1}\right)$. Let us denote this kind of symbols by $N_{n}$. Although $T_{N_{n}}$ is commutative, it is too large, so it is impossible to figure out what its spectrum is. In particular, in $[21,22]$, the authors described the algebra generated by Toeplitz operators acting on the weighted Bergman space $\mathscr{A}_{\lambda}^{2}\left(D_{3}\right)$ over threedimensional Siegel domain $D_{3}$ using nilpotent symbols of the form $c\left(y_{2}\right) g\left(\operatorname{Im} \zeta_{3}-\left|\left(\zeta_{1}, \zeta_{2}\right)\right|^{2}\right)$.

The main purpose of the paper is to find the spectrum of the algebra generated by Toeplitz operators acting on the true-poly-Bergman type space $\mathscr{A}_{(L)}^{2}\left(D_{2}\right)$ over twodimensional Siegel domain $D_{2}$ by selecting a particular set of nilpotent symbols. In this sense, we just consider nilpotent symbols of the form $a\left(\operatorname{Im} \zeta_{1}\right)$ and $b\left(\operatorname{Im} \zeta_{2}-\left|\zeta_{1}\right|^{2}\right)$. This paper is organized as follows. In Section 2, we recall how poly-Bergman type spaces are defined for the Siegel domain, and how they can be identified with a $L^{2}$-space through a Bargmann type transform. In Section 3, we introduce Toeplitz operators acting on $\mathscr{A}_{(L)}^{2}\left(D_{2}\right)$ with nilpotent symbols; we show that such Toeplitz operators are unitary equivalent to multiplication operators. In Section 4, we take symbols of the form $b\left(\operatorname{Im} \zeta_{2}-\left|\zeta_{1}\right|^{2}\right)$ for which both limits $\lim _{s \rightarrow 0^{+}} b(s)$ and $\lim _{s \rightarrow+\infty} b(s)$ exist; it is proved that the $C^{*}$-algebra generated by all Toeplitz operators $T_{b}$ is isomorphic to $C\left(\overline{\mathbb{R}}_{+}\right)$, where $\overline{\mathbb{R}}_{+}=[0,+\infty]$ is the one-point compactification of $[0,+\infty)$. In Section 5, we take nilpotent symbols of the form $a\left(\operatorname{Im} \zeta_{1}\right)$, where $a \in C(\overline{\mathbb{R}})$, and $\overline{\mathbb{R}}=[-\infty,+\infty]$ is the two-point compactification of $\mathbb{R}$; we prove that the $C^{*}$-algebra generated by all Toeplitz operators $T_{a}$ is isomorphic to $C(\Delta)$, where $\Delta=\bar{\Pi}$ $/(\overline{\mathbb{R}} \times\{+\infty\})$ and $\bar{\Pi}=\overline{\mathbb{R}} \times \overline{\mathbb{R}}_{+}$. In Section 6, we describe the $C^{*}$-algebra generated by all Toeplitz operators $T_{d}$, where $d\left(\operatorname{Im} \zeta_{1}\right) \in P C(\overline{\mathbb{R}}, S)$, and $P C(\overline{\mathbb{R}}, S)$ is the set of all piecewise continuous functions on $\overline{\mathbb{R}}$ having one-side limit values at each point of a finite set $S \subset \mathbb{R}$. Finally, in Section 7 , we describe the $C^{*}$-algebra generated by all Toeplitz operators $T_{a b}=T_{a} T_{b}=T_{b} T_{a}$.

\section{Poly-Bergman Type Spaces of the Siegel Domain}

In this section, we recall some results obtained in [23], which are needed in our research about Toeplitz operators. Each $\zeta$ $\in \mathbb{C}^{n}$ will be represented as an ordered pair $\zeta=\left(\zeta^{\prime}, \zeta_{n}\right)$, where $\zeta^{\prime}=\left(\zeta_{1}, \cdots, \zeta_{n-1}\right) \in \mathbb{C}^{n-1}$. Besides, the Euclidean norm function will be denoted by $|\cdot|$. The Siegel domain is defined by

$$
D_{n}=\left\{\zeta=\left(\zeta^{\prime}, \zeta_{n}\right) \in \mathbb{C}^{n-1} \times \mathbb{C}: \operatorname{Im} \zeta_{n}-\left|\zeta^{\prime}\right|^{2}>0\right\}
$$

We will study Toeplitz operators acting on certain poly-Bergman type subspaces of $L^{2}\left(D_{n}, d \mu_{\lambda}\right)$, where $d$ $\mu_{\lambda}(\zeta)=\left(\operatorname{Im} \zeta_{n}-\left|\zeta^{\prime}\right|^{2}\right)^{\lambda} d \mu(\zeta)$, with $\lambda>-1$, and $d \mu(\zeta)$ is the usual Lebesgue measure. Once and for all, $L^{2}(X)$ means $L^{2}(X, d m)$, where $X$ is any subset of a Euclidean space and $d m$ is the Lebesgue area measure on $X$.

For each multi-index $L=\left(l_{1}, \cdots, l_{n}\right) \in \mathbb{N}^{n}$, the polyBergman type space $\mathscr{A}_{\lambda L}^{2}\left(D_{n}\right)$ is the closed subspace of $L^{2}\left(D_{n}, d \mu_{\lambda}\right)$ consisting of all $L$-analytic functions, that is, all functions $f(\zeta)$ satisfying the equations

$$
\begin{gathered}
\left(\frac{\partial}{\partial \bar{\zeta}_{m}}-2 i \zeta_{m} \frac{\partial}{\partial \bar{\zeta}_{n}}\right)^{l_{m}} f=0, \quad 1 \leq m \leq n-1, \\
\left(\frac{\partial}{\partial \bar{\zeta}_{n}}\right)^{l_{n}} f=0 .
\end{gathered}
$$

In particular, for $L=(1, \cdots, 1), \mathscr{A}_{\lambda L}^{2}\left(D_{n}\right)$ is just the Bergman space. Likewise, the anti-poly-Bergman type space $\tilde{\mathscr{A}}_{\lambda L}^{2}\left(D_{n}\right)$ is defined to be the complex conjugate of $\mathscr{A}_{\lambda L}^{2}\left(D_{n}\right)$. Thus, we introduce true-poly-Bergman type spaces as follows:

$$
\begin{aligned}
& \mathscr{A}_{\lambda(L)}^{2}\left(D_{n}\right)=\mathscr{A}_{\lambda L}^{2}\left(D_{n}\right) \ominus\left(\sum_{m=1}^{n} \mathscr{A}_{\lambda, L-e_{m}}^{2}\left(D_{n}\right)\right), \\
& \tilde{\mathscr{A}}_{\lambda(L)}^{2}\left(D_{n}\right)=\tilde{\mathscr{A}}_{\lambda L}^{2}\left(D_{n}\right) \ominus\left(\sum_{m=1}^{n} \tilde{\mathscr{A}}_{\lambda, L-e_{m}}^{2}\left(D_{n}\right)\right),
\end{aligned}
$$

where $e_{m}=(0, \cdots, 1, \cdots, 0)$ and the 1 is placed at the $m$ -entry. We assume that $\mathscr{A}_{\lambda(L)}^{2}\left(D_{n}\right)=\{0\}$ whenever $L \epsilon$ $\mathbb{Z}^{n} \backslash \mathbb{N}^{n}$.

In [23], the authors proved that $L^{2}\left(D_{n}, d \mu_{\lambda}\right)$ equals to the direct sum of all the true-poly-Bergman type spaces:

$$
L^{2}\left(D_{n}, d \mu_{\lambda}\right)=\left(\underset{L \in \mathbb{N}^{n}}{\oplus} \mathscr{A}_{\lambda(L)}^{2}\left(D_{n}\right)\right) \oplus\left(\underset{L \in \mathbb{N}^{n}}{\oplus} \tilde{A}_{\lambda(L)}^{2}\left(D_{n}\right)\right) .
$$

The authors also proved that $\mathscr{A}_{\lambda(L)}^{2}\left(D_{n}\right)$ is isomorphic and isometric to the tensor product

$$
L^{2}\left(\mathbb{R}^{n-1}\right) \otimes \mathbb{H}_{l_{1}-1} \otimes \cdots \otimes \mathbb{H}_{l_{n-1}-1} \otimes L^{2}\left(\mathbb{R}_{+}\right) \otimes \mathscr{L}_{l_{n}-1},
$$

where $\mathbb{R}_{+}=(0, \infty)$. Both $\mathbb{H}_{m}$ and $\mathscr{L}_{m}$ are onedimensional spaces defined below. Recall the Hermite and Laguerre polynomials:

$$
H_{m}(y):=(-1)^{m} e^{y^{2}} \frac{d^{m}}{d y^{m}}\left(e^{-y^{2}}\right), L_{m}^{\lambda}(y):=e^{y} \frac{y^{-\lambda}}{m !} \frac{d^{m}}{d y^{m}}\left(e^{-y} y^{m+\lambda}\right),
$$


for $m=0,1,2, \cdots$ Recall also the Hermite and Laguerre functions

$h_{m}(y)=\frac{(-1)^{m}}{\left(2^{n} \sqrt{\pi} n !\right)^{1 / 2}} H_{m}(y) e^{-y^{2} / 2}, \ell_{m}^{\lambda}(y)=(-1)^{m} c_{m} L_{m}^{\lambda}(y) e^{-y / 2}$,

where $c_{m}=\sqrt{m ! / \Gamma(m+\lambda+1)}$ and $\Gamma$ is the usual Gamma function. It is well known that $\left\{h_{m}\right\}_{m=0}^{\infty}$ and $\left\{\ell_{m}^{\lambda}\right\}_{m=0}^{\infty}$ are orthonormal bases for $L^{2}(\mathbb{R})$ and $L^{2}\left(\mathbb{R}_{+}\right.$, $\left.y^{\lambda} d y\right)$, respectively. Finally, $\mathbb{H}_{m}=\operatorname{span}\left\{h_{m}\right\}$ and $\mathscr{L}_{m}=$ $\operatorname{span}\left\{\ell_{m}^{\lambda}\right\}$.

In this work, we restrict ourselves to the study of Toeplitz operators acting on the true-poly-Bergman type spaces over two-dimensional Siegel domain $D_{2}$ with the Lebesgue measure $d \mu(\lambda=0)$. Henceforth, the space $\mathscr{A}_{0(L)}^{2}\left(D_{2}\right)$ will be simply denoted by $\mathscr{A}_{(L)}^{2}\left(D_{2}\right)$; similarly, $\ell_{m}(y)$ and $L_{m}(y)$ stand for $\ell_{m}^{0}(y)$ and $L_{m}^{0}(y)$, respectively. The true-poly-Bergman type space $\mathscr{A}_{(L)}^{2}\left(D_{2}\right)$ can be identified with $L^{2}\left(\mathbb{R} \times \mathbb{R}_{+}\right)$ through a Bargmann type transform ([23]), such identification fits to the study of Toeplitz operators with nilpotent symbols. Several operators are needed to define such identification. To begin with, we introduce the flat domain $\mathscr{D}=\mathbb{C}$ $\times \Pi$, where $\Pi=\mathbb{R} \times \mathbb{R}_{+} \subset \mathbb{C}$. Then, $\mathscr{D}$ can be identified with $D_{2}$ using the mapping

$$
\kappa: \mathscr{D} \ni w=\left(w_{1}, w_{2}\right) \mapsto \zeta=\left(w_{1}, w_{2}+i\left|w_{1}\right|^{2}\right) \in D_{2}
$$

Thus, we have the unitary operator $U_{0}: L^{2}\left(D_{2}, d \mu\right)$ $\longrightarrow L^{2}(\mathscr{D}, d \eta)$ given by

$$
\left(U_{0} f\right)(w)=f(\kappa(w))
$$

where $d \eta(w)=d \mu(w)$. Take $w=\left(w_{1}, w_{2}\right) \in \mathbb{C} \times \Pi$, with $w_{m}$ $=u_{m}+i v_{m}$ and $m=1,2$. We identify $w=\left(u_{1}+i v_{1}, u_{2}+i v_{2}\right)$ with $\left(u_{1}, v_{1}, u_{2}, v_{2}\right)$. Then

$L^{2}(\mathscr{D}, d \eta)=L^{2}\left(\mathbb{R}, d u_{1}\right) \otimes L^{2}\left(\mathbb{R}, d v_{1}\right) \otimes L^{2}\left(\mathbb{R}, d u_{2}\right) \otimes L^{2}\left(\mathbb{R}_{+}, d v_{2}\right)$.

Introduce

$$
U_{1}=F \otimes I \otimes F \otimes I,
$$

where $F$ is the Fourier transform acting on $L^{2}(\mathbb{R})$ by the rule

$$
(F g)(t)=\frac{1}{\sqrt{2 \pi}} \int_{-\infty}^{\infty} g(x) e^{-i t x} d x
$$

Consider now the following two mappings acting on $\mathscr{D}$ :

$$
\begin{aligned}
\psi_{1}: \xi & =\left(\xi_{1}, t_{2}+i s_{2}\right) \mapsto w=\left(\xi_{1}, t_{2}+i \frac{s_{2}}{2\left|t_{2}\right|}\right), \\
\psi_{2}: z & =\left(x_{1}+i y_{1}, z_{2}\right) \mapsto \xi \\
& =\left(\sqrt{\left|x_{2}\right|}\left(x_{1}+y_{1}\right)+i \frac{1}{2 \sqrt{\left|x_{2}\right|}}\left(-x_{1}+y_{1}\right), z_{2}\right),
\end{aligned}
$$

where $\xi=\left(\xi_{1}, \xi_{2}\right), z=\left(z_{1}, z_{2}\right) \in \mathscr{D}, \xi_{m}=t_{m}+i s_{m}$, and $z_{m}=$ $x_{m}+i y_{m}$. Both functions $\psi_{1}$ and $\psi_{2}$ lead to the following unitary operators acting on $L^{2}(\mathscr{D}, d \eta)$ :

$$
\left(V_{1} f\right)(\xi)=\frac{1}{\left(2\left|t_{2}\right|\right)^{1 / 2}} f\left(\psi_{1}(\xi)\right),\left(V_{2} g\right)(z)=g\left(\psi_{2}(z)\right) .
$$

Henceforth, $L=\left(l_{1}, l_{2}\right)=(j, k)$.

Theorem 1 (see [23]). The operator $U=V_{2} V_{1} U_{1} U_{0}$ is unitary and maps $L^{2}\left(D_{2}, d \mu\right)$ onto the space

$L^{2}(\mathscr{D}, d \eta)=L^{2}\left(\mathbb{R}, d x_{1}\right) \otimes L^{2}\left(\mathbb{R}, d y_{1}\right) \otimes L^{2}\left(\mathbb{R}, d x_{2}\right) \otimes L^{2}\left(\mathbb{R}_{+}, d y_{2}\right)$

For each $L=(j, k) \in \mathbb{N}^{2}$, the operator $U$ restricted to $\mathscr{A}_{(L)}^{2}\left(D_{2}\right)$ is an isometric isomorphism onto the space

$$
\mathscr{H}_{(L)}^{+}=L^{2}(\mathbb{R}) \otimes \operatorname{span}\left\{h_{j-1}\left(y_{1}\right)\right\} \otimes L^{2}\left(\mathbb{R}_{+}\right) \otimes \operatorname{span}\left\{\ell_{k-1}\left(y_{2}\right)\right\}
$$

Introduce the isometric linear embedding $R_{0(L)}: L^{2}$ $\left(\mathbb{R} \times \mathbb{R}_{+}\right) \longrightarrow L^{2}(\mathscr{D})$ defined by

$$
\left(R_{0(L)} g\right)\left(x_{1}, y_{1}, x_{2}, y_{2}\right)=\chi_{\mathbb{R}_{+}}\left(x_{2}\right) g\left(x_{1}, x_{2}\right) h_{j-1}\left(y_{1}\right) \ell_{k-1}\left(y_{2}\right) .
$$

Of course, $\mathscr{H}_{(L)}^{+}$is the range of $R_{0(L)}$, and it is also the image of $\mathscr{A}_{(L)}^{2}\left(D_{2}\right)$ under $U$. Thus, the operator

$$
R_{(L)}=R_{0(L)}^{*} U: L^{2}\left(D_{2}\right) \longrightarrow L^{2}\left(\mathbb{R} \times \mathbb{R}_{+}\right),
$$

isometrically maps the true-poly-Bergman type space $\mathscr{A}_{(L)}^{2}$ $\left(D_{2}\right)$ onto $L^{2}\left(\mathbb{R} \times \mathbb{R}_{+}\right)$. Therefore, $R_{(L)} R_{(L)}^{*}=I$ and $R_{(L)}^{*} R_{(L)}$ $=B_{(L)}$, where $B_{(L)}$ is the orthogonal projection from $L^{2}\left(D_{2}\right)$ onto $\mathscr{A}_{(L)}^{2}\left(D_{2}\right)$. In addition, the operator $R_{(L)}^{*}=U^{*} R_{0(L)}$ plays the role of the Segal-Bargmann transform for the true-polyBergman type space $\mathscr{A}_{(L)}^{2}\left(D_{2}\right)$, where the adjoint operator $R_{0(L)}^{*}: L^{2}(\mathscr{D}) \longrightarrow L^{2}\left(\mathbb{R} \times \mathbb{R}_{+}\right)$is given by 


$$
\left(R_{0(L)}^{*} f\right)\left(x_{1}, x_{2}\right)=\int_{\mathbb{R}} \int_{\mathbb{R}_{+}} h_{j-1}\left(y_{1}\right) \ell_{k-1}\left(y_{2}\right) f\left(x_{1}, y_{1}, x_{2}, y_{2}\right) d y_{2} d y_{1},
$$

with $\left(x_{1}, x_{2}\right) \in \mathbb{R} \times \mathbb{R}_{+}$.

\section{Toeplitz Operators with Nilpotent Symbols}

In this section, we study Toeplitz operators with nilpotent symbols and acting on the true-poly-Bergman type space $\mathscr{A}_{(L)}^{2}\left(D_{2}\right)$. In [3], the author has widely developed the theory of Toeplitz operators on the Bergman spaces, and the author's techniques can be applied to the study of Toeplitz operators acting on $\mathscr{A}_{(L)}^{2}\left(D_{2}\right)$. To begin with, a function $c \in L^{\infty}\left(D_{2}, d \mu\right)$ is said to be a nilpotent symbol if it has the form $c\left(\zeta_{1}, \zeta_{2}\right)=c(\operatorname{Im}$ $\left.\zeta_{1}, \operatorname{Im} \zeta_{2}-\left|\zeta_{1}\right|^{2}\right)$. Then, the Toeplitz operator acting on $\mathscr{A}_{(L)}^{2}\left(D_{2}\right)$, with nilpotent symbol $c(\zeta)$, is defined by

$$
\left(T_{c} f\right)(\zeta)=\left(B_{(L)}(c f)\right)(\zeta)
$$

where $B_{(L)}$ is the orthogonal projection from $L^{2}\left(D_{2}\right)$ onto $\mathscr{A}_{(L)}^{2}\left(D_{2}\right)$. The Bargmann-type operator $R_{(L)}$ identifies the space $\mathscr{A}_{(L)}^{2}\left(D_{2}\right)$ with $L^{2}\left(\mathbb{R} \times \mathbb{R}_{+}\right)$, and it fits properly in the study of the Toeplitz operator $T_{c}$.

Theorem 2. Let $c$ be a nilpotent symbol. Then, the Toeplitz operator $T_{c}$ is unitary equivalent to the multiplication operator $\gamma^{c} I=R_{(L)} T_{c} R_{(L)}^{*}$, where $\gamma^{c}: \mathbb{R} \times \mathbb{R}_{+} \longrightarrow \mathbb{C}$ is given by

$\gamma^{c}\left(x_{1}, x_{2}\right)=\int_{\mathbb{R}} \int_{\mathbb{R}_{+}} c\left(\frac{-x_{1}+y_{1}}{2 \sqrt{x_{2}}}, \frac{y_{2}}{2 x_{2}}\right)\left(h_{j-1}\left(y_{1}\right)\right)^{2}\left(\ell_{k-1}\left(y_{2}\right)\right)^{2} d y_{2} d y_{1}$.

Proof. We have

$$
\begin{aligned}
R_{(L)} T_{c} R_{(L)}^{*} & =R_{(L)} B_{(L)}(c I) R_{(L)}^{*} \\
& =R_{(L)} R_{(L)}^{*} R_{(L)}(c I) R_{(L)}^{*} \\
& =R_{(L)}(c I) R_{(L)}^{*} \\
& =R_{0(L)}^{*} V_{2} V_{1} U_{1} U_{0}(c I) U_{0}^{-1} U_{1}^{-1} V_{1}^{-1} V_{2}^{-1} R_{0(L)} .
\end{aligned}
$$

Recall that $\zeta=\kappa(w)=\left(w_{1}, w_{2}+i\left|w_{1}\right|^{2}\right)$, where $w=\left(w_{1}\right.$, $\left.w_{2}\right) \in \mathscr{D}$ and $w_{m}=u_{m}+i v_{m}$. For $g \in L^{2}(\mathscr{D})$,

$$
\left(U_{0}(c I) U_{0}^{-1} g\right)(w)=c(\kappa(w))\left(U_{0}^{-1} g\right)(\kappa(w))=c(\kappa(w)) g(w) .
$$

That is, $U_{0}(c I) U_{0}^{-1}=c(\kappa(w)) I$, where $c(\kappa(w))=c\left(v_{1}, v_{2}\right)$.
It is easy to see that $U_{1}\left(c\left(v_{1}, v_{2}\right) I\right) U_{1}^{-1}=c\left(v_{1}, v_{2}\right) I$,

$$
\begin{gathered}
V_{1}(c I) V_{1}^{-1}=c\left(s_{1}, \frac{s_{2}}{2\left|t_{2}\right|}\right) I, \\
V_{2} V_{1}(c I) V_{1}^{-1} V_{2}^{-1}=c\left(\frac{-x_{1}+y_{1}}{2 \sqrt{\left|x_{2}\right|}}, \frac{y_{2}}{2\left|x_{2}\right|}\right) I .
\end{gathered}
$$

Thus

$$
R_{(L)} T_{c} R_{(L)}^{*}=R_{0(L)}^{*} c\left(\frac{-x_{1}+y_{1}}{2 \sqrt{\left|x_{2}\right|}}, \frac{y_{2}}{2\left|x_{2}\right|}\right) I R_{0(L)}=\gamma^{c}\left(x_{1}, x_{2}\right) I,
$$

where $\gamma^{c}\left(x_{1}, x_{2}\right)$ is given in (21).

By Theorem 2, the $C^{*}$-algebra generated by all Toeplitz operators $T_{c}$ is commutative (see $[1,3]$ ), but its spectrum is difficult to figure out what it is. For this reason, we assume certain continuity conditions on the nilpotent symbols in order to describe the spectrum of the subalgebra generated by the Toeplitz operators. We will split our research into two cases concerning the symbols. Firstly, we study Toeplitz operators with symbols of the form $b\left(\operatorname{Im} \zeta_{2}-\left|\zeta_{1}\right|^{2}\right)$, for which

$$
\gamma^{b}\left(x_{1}, x_{2}\right)=\gamma^{b}\left(x_{2}\right)=\int_{\mathbb{R}_{+}} b\left(\frac{y_{2}}{2 x_{2}}\right)\left(\ell_{k-1}\left(y_{2}\right)\right)^{2} d y_{2} .
$$

Secondly, we analyze Toeplitz operators with symbols of the form $a\left(\operatorname{Im} \zeta_{1}\right)$, for which

$$
\gamma^{a}\left(x_{1}, x_{2}\right)=\int_{\mathbb{R}} a\left(\frac{-x_{1}+y_{1}}{2 \sqrt{x_{2}}}\right)\left(h_{j-1}\left(y_{1}\right)\right)^{2} d y_{1} .
$$

As mentioned above, the $C^{*}$-algebra generated by all Toeplitz operators $T_{a}$ is still complicated to be fully described despite its commutative property. Fortunately, the $C^{*}$-algebra generated by all operators $T_{a}$ can be described when the symbols $a$ are taken to be continuous on $\overline{\mathbb{R}}=[-\infty,+\infty]$, where $\overline{\mathbb{R}}$ is the two-point compactification of $\mathbb{R}$. Even more, the $C^{*}$-algebra of Toeplitz operators $T_{a}$ can be still described for symbols having finitely many jump discontinuities, as shown in Section 6. Finally, we analyze Toeplitz operators with symbols of the form $c\left(\zeta_{1}, \zeta_{2}\right)=a\left(\operatorname{Im} \zeta_{1}\right) b\left(\operatorname{Im} \zeta_{2}-\left|\zeta_{1}\right|^{2}\right)$.

\section{Toeplitz Operators with \\ Symbols $b\left(\operatorname{Im} \zeta_{2}-\left|\zeta_{1}\right|^{2}\right)$}

In this section, we study the $C^{*}$-algebra generated by all Toeplitz operators $T_{b}$ with symbols of the form $b\left(\operatorname{Im} \zeta_{2}-\left|\zeta_{1}\right|^{2}\right)$, where $b(y)$ has limit values at $y=0,+\infty$. Under this continuity condition, we will see that $\gamma^{b}$ is continuous on $\bar{\Pi}:=\overline{\mathbb{R}} \times$ $\overline{\mathbb{R}}_{+}$, where $\overline{\mathbb{R}}_{+}=[0,+\infty]$ is the two-point compactification of $\mathbb{R}_{+}=(0,+\infty)$. Apply the change of variable $y_{2} \mapsto 2 x_{2} y_{2}$ in the integral representation of $\gamma^{b}$, then 


$$
\gamma^{b}\left(x_{1}, x_{2}\right)=\gamma^{b}\left(x_{2}\right)=2 x_{2} \int_{\mathbb{R}_{+}} b\left(y_{2}\right)\left(\ell_{k-1}\left(2 x_{2} y_{2}\right)\right)^{2} d y_{2} .
$$

Actually, $\gamma^{b}$ depends only on the variable $x_{2}$ and is continuous on $\mathbb{R}_{+}$because of the continuity of $\ell_{k-1}(y)$ and the Lebesgue dominated convergence theorem.

Let $L_{\{0,+\infty\}}^{\infty}\left(\mathbb{R}_{+}\right)$denote the subspace of $L^{\infty}\left(\mathbb{R}_{+}\right)$consisting of all functions having limit values at 0 and $+\infty$. For $b$ $\in L_{\{0,+\infty\}}^{\infty}\left(\mathbb{R}_{+}\right)$, define

$$
b_{0}:=\lim _{y \rightarrow 0^{+}} b(y), b_{\infty}:=\lim _{y \rightarrow+\infty} b(y) .
$$

It is worth mentioning that $\gamma^{b}$ was obtained in [10] as the spectral function of a Toeplitz operator acting on a true-polyBergman space of the upper half-plane. Thus, we have at least two scenarios in which $\gamma^{b}$ appears as a spectral function.

Lemma 3 (see [10]). Let $b \in L_{\{0,+\infty\}}^{\infty}\left(\mathbb{R}_{+}\right)$. Then, the spectral function $\gamma^{b}$ satisfies

$$
b_{\infty}=\lim _{x_{2} \rightarrow 0^{+}} \gamma^{b}\left(x_{2}\right), \quad b_{0}=\lim _{x_{2} \rightarrow+\infty} \gamma^{b}\left(x_{2}\right) .
$$

According to Lemma 3 and Theorem 4.8 in [10], we have the following.

Theorem 4. For $b \in L_{\{0,+\infty\}}^{\infty}\left(\mathbb{R}_{+}\right)$, the spectral function $\gamma^{b}\left(x_{2}\right)$ is continuous on $\overline{\mathbb{R}}=[0,+\infty]$. The $C^{*}$-algebra generated by all functions $\gamma^{b}$, with $b \in L_{\{0,+\infty\}}^{\infty}\left(\mathbb{R}_{+}\right)$, is isomorphic and isometric to the algebra $C[0, \infty]$. That is, the $C^{*}$-algebra generated by all Toeplitz operators $T_{b}$, with $b\left(\operatorname{Im} \zeta_{2}-\right.$ $\left.\left|\zeta_{1}\right|^{2}\right) \in L_{\{0,+\infty\}}^{\infty}\left(\mathbb{R}_{+}\right)$, is isomorphic to $C[0, \infty]$, where the isomorphism is defined on the generators by

$$
T_{b} \mapsto \gamma^{b}
$$

Obviously, the spectral function $\gamma^{b}\left(x_{2}\right)$ is defined and continuous on $\bar{\Pi}$, but it is constant along each horizontal straight line. Thus, $\gamma^{b}$ is identified with a continuous function on the quotient space $\bar{\Pi} / \overline{\mathbb{R}}$, which is homeomorphic to $\overline{\mathbb{R}}_{+}$.

\section{Toeplitz Operators with Continuous Symbols $a\left(\operatorname{Im} \zeta_{1}\right)$}

In this section, we study the $C^{*}$-algebra generated by all Toeplitz operators $T_{a}$, where symbols $a\left(\operatorname{Im} \zeta_{1}\right)$ are taken to be continuous on $\overline{\mathbb{R}}$. Once again, such a $C^{*}$-algebra can be identified with the algebra of all continuous functions on a quotient space of $\bar{\Pi}$. Henceforth, $\left(x_{1}, x_{2}\right)$ will denote points in $\bar{\Pi}$ instead of intervals.

It is fairly simple to see that $\gamma^{a}$ is continuous on $\Pi$. Take the change of variable $y_{1} \mapsto 2 \sqrt{x_{2}} y_{1}+x_{1}$ in the integral representation of $\gamma^{a}$, then

$$
\gamma^{a}\left(x_{1}, x_{2}\right)=2 \sqrt{x_{2}} \int_{\mathbb{R}} a\left(y_{1}\right)\left(h_{j-1}\left(2 \sqrt{x_{2}} y_{1}+x_{1}\right)\right)^{2} d y_{1} .
$$

The function $\gamma^{a}$ is continuous at each point $\left(x_{1}, x_{2}\right) \in \Pi$ because of the continuity of $h_{j-1}$ and the Lebesgue dominated convergence theorem. Next, we will prove that $\gamma^{a}$ has oneside limit value at each point of $\mathbb{R} \times\{0\}$. For $a \in L^{\infty}(\mathbb{R})$, we introduce the notation

$$
a_{-}=\lim _{y \rightarrow-\infty} a(y) \text { and } a_{+}=\lim _{y \rightarrow+\infty} a(y),
$$

if such limits exist.

Lemma 5. Let $a \in L^{\infty}(\mathbb{R})$, and suppose that a $(y)$ converges at $y= \pm \infty$. Then, for each $x_{0} \in \mathbb{R}$, the spectral function $\gamma^{a}$ satisfies

$\lim _{\left(x_{1}, x_{2}\right) \rightarrow\left(x_{0}, 0\right)} \gamma^{a}\left(x_{1}, x_{2}\right)=a_{-} \int_{-\infty}^{x_{0}}\left(h_{j-1}\left(y_{1}\right)\right)^{2} d y_{1}+a_{+} \int_{x_{0}}^{\infty}\left(h_{j-1}\left(y_{1}\right)\right)^{2} d y_{1}$.

Proof. Let $A$ denote the right-hand side of equality (34). Take $\varepsilon>0$. We will prove that there exist $\delta>0$ such that $\mid \gamma^{a}\left(x_{1}\right.$, $\left.x_{2}\right)-A \mid<\varepsilon$ whenever $\left|x_{1}-x_{0}\right|<\delta$ and $0<x_{2}<\delta$. Note that $\left|a_{-}\right|,\left|a_{+}\right| \leq\|a\|_{\infty}$. Since $\int_{-\infty}^{\infty}\left(h_{j-1}\left(y_{1}\right)\right)^{2} d y_{1}=1$, there exists $\delta_{1}>0$ such that

$$
\|a\|_{\infty} \int_{-\delta_{1}+x_{0}}^{\delta_{1}+x_{0}}\left(h_{j-1}\left(y_{1}\right)\right)^{2} d y_{1}<\frac{\varepsilon}{5} .
$$

Then

$$
\begin{aligned}
I:= & \left|\gamma^{a}\left(x_{1}, x_{2}\right)-A\right| \\
= & \mid \int_{-\infty}^{\infty} a\left(\frac{-x_{1}+y_{1}}{2 \sqrt{x_{2}}}\right)\left(h_{j-1}\left(y_{1}\right)\right)^{2} d y_{1}-a_{-} \int_{-\infty}^{x_{0}}\left(h_{j-1}\left(y_{1}\right)\right)^{2} d y_{1} \\
& -a_{+} \int_{x_{0}}^{\infty}\left(h_{j-1}\left(y_{1}\right)\right)^{2} d y_{1} \mid \\
\leq & \int_{-\infty}^{-\delta_{1}+x_{0}}\left|a\left(\frac{-x_{1}+y_{1}}{2 \sqrt{x_{2}}}\right)-a_{-}\right|\left(h_{j-1}\left(y_{1}\right)\right)^{2} d y_{1} \\
& +\left|a_{-}\right| \int_{-\delta_{1}+x_{0}}^{x_{0}}\left(h_{j-1}\left(y_{1}\right)\right)^{2} d y_{1}+\left|a_{+}\right| \int_{x_{0}}^{\delta_{1}+x_{0}}\left(h_{j-1}\left(y_{1}\right)\right)^{2} d y_{1} \\
& +\int_{-\delta_{1}+x_{0}}^{\delta_{1}+x_{0}}\left|a\left(\frac{-x_{1}+y_{1}}{2 \sqrt{x_{2}}}\right)\left(h_{j-1}\left(y_{1}\right)\right)^{2}\right| d y_{1} \\
& +\int_{\delta_{1}+x_{0}}^{\infty}\left|a\left(\frac{-x_{1}+y_{1}}{2 \sqrt{x_{2}}}\right)-a_{+}\right|\left(h_{j-1}\left(y_{1}\right)\right)^{2} d y_{1} \\
\leq & \max _{-\infty<y_{1}<-\delta_{1}+x_{0}}\left|a\left(\frac{-x_{1}+y_{1}}{2 \sqrt{x_{2}}}\right)-a_{-}\right|+\frac{3 \varepsilon}{5}+\max _{\delta_{1}+x_{0}<y_{1}<\infty} \\
& \cdot\left|a\left(\frac{-x_{1}+y_{1}}{2 \sqrt{x_{2}}}\right)-a_{+}\right| .
\end{aligned}
$$


We have assumed that $a(y)$ converges at $\pm \infty$; then, there exists $N>0$ such that $\left|a(y)-a_{-}\right|<\varepsilon / 5$ and $\left|a(y)-a_{+}\right|<\varepsilon / 5$ for $|y|>N$. Let $\delta=\min \left\{\delta_{1} / 2, \delta_{1}^{2} /\left(16 N^{2}\right)\right\}$. Then, we have 1 $/\left(2 \sqrt{x_{2}}\right)\left|-x_{1}+y_{1}\right|>N$ if $\left|x_{1}-x_{0}\right|<\delta, 0<x_{2}<\delta$, and $\mid y_{1}-$ $x_{0} \mid \geq \delta_{1}$. Thus,

$$
\begin{gathered}
\max _{-\infty<y_{1}<-\delta_{1}+x_{0}}\left|a\left(\frac{-x_{1}+y_{1}}{2 \sqrt{x_{2}}}\right)-a_{-}\right|<\frac{\varepsilon}{5}, \\
\max _{\delta_{1}+x_{0}<y_{1}<\infty}\left|a\left(\frac{-x_{1}+y_{1}}{2 \sqrt{x_{2}}}\right)-a_{+}\right|<\frac{\varepsilon}{5} .
\end{gathered}
$$

Finally, we conclude that $\left|\gamma^{a}\left(x_{1}, x_{2}\right)-A\right|<\varepsilon$ whenever $\mid$ $x_{1}-x_{0} \mid<\delta$ and $0<x_{2}<\delta$.

In general, $\gamma^{a}\left(x_{1}, x_{2}\right)$ does not converge at each point $x$ $=( \pm \infty,+\infty) \in \bar{\Pi}$; however, $\gamma^{a}\left(x_{1}, x_{2}\right)$ has limit values along the parabolas $x_{2}=\alpha\left(x_{1}^{2}+1\right)$, with $\alpha>0$. We will define a bijective mapping $\Phi: \Pi \longrightarrow \Pi$ so that $\phi^{a}=\gamma^{a} \circ \Phi^{-1}$ will be a continuous mapping on $\bar{\Pi}=\overline{\mathbb{R}} \times \overline{\mathbb{R}}_{+}$with the usual topology.

5.1. Modified Spectral Function for $T_{a}$. Let $\Phi: \Pi \longrightarrow \Pi$ be the mapping

$$
\Phi\left(x_{1}, x_{2}\right)=\left(x_{1}, \frac{x_{2}}{x_{1}^{2}+1}\right) .
$$

It is easy to see that $\Phi^{-1}\left(t_{1}, t_{2}\right)=\left(t_{1},\left(t_{1}^{2}+1\right) t_{2}\right)$. Concerning the spectral properties of $T_{a}$, the function $\phi^{a}:=\gamma^{a}$ 。 $\Phi^{-1}$ is as important as $\gamma^{a}$, but $\phi^{a}$ behaves much better than $\gamma^{a}$, at least for a continuous on $\overline{\mathbb{R}}$. From now on, we take $\phi^{a}$ as the spectral function for the Toeplitz operator $T_{a}$. A direct computation shows that

$$
\phi^{a}\left(t_{1}, t_{2}\right)=\int_{-\infty}^{\infty} a\left(\frac{-t_{1}+s_{1}}{2 \sqrt{t_{2}\left(t_{1}^{2}+1\right)}}\right)\left(h_{j-1}\left(s_{1}\right)\right)^{2} d s_{1} .
$$

Both $\Phi$ and $\Phi^{-1}$ are continuous on $\Pi$. Besides, the spectral function $\phi^{a}=\gamma^{a} \circ \Phi^{-1}$ is continuous on $\Pi$ because $\gamma^{a}$ is. Since $\Phi^{-1}\left(t_{1}, 0\right)=\left(t_{1}, 0\right)$, we have $\phi^{a}\left(t_{1}, 0\right)=\gamma^{a}\left(x_{1}, 0\right)$. By Lemma $5, \phi^{a}$ is also continuous on $\mathbb{R} \times\{0\}$.

Theorem 6. For $a\left(\operatorname{Im} \zeta_{1}\right) \in C(\overline{\mathbb{R}})$, the spectral function $\phi^{a}: \Pi \longrightarrow \mathbb{C}$ can be extended continuously to $\bar{\Pi}=\overline{\mathbb{R}} \times$ $\overline{\mathbb{R}}_{+}$.

Proof. Follows from Lemmas 5 and 7-9.

For any domain $X \subset \mathbb{R}^{m}$ and a function $\varphi: X \longrightarrow \mathbb{C}$, we write $\varphi\left(x_{0}\right)$ to mean the limit value of $\varphi$ at $x_{0}$, even if $x_{0}$ does not belong to $X$. For example, $a(+\infty)$ means $\lim _{x \rightarrow+\infty} a(x)$.
Lemma 7. Let $a \in L^{\infty}(\mathbb{R})$, and suppose that $a(y)$ converges at the points $y= \pm \infty$. Then, $\phi^{a}$ satisfies

$$
\lim _{\left(t_{1}, t_{2}\right) \rightarrow(+\infty, 0)} \phi^{a}\left(t_{1}, t_{2}\right)=a(-\infty) .
$$

That is, for $\varepsilon>0$, there exists $\delta>0$ and $N>0$ such that $\left|\phi^{a}\left(t_{1}, t_{2}\right)\right|<\varepsilon$ whenever $0<t_{2}<\delta$ and $t_{1}>N$. Analogously,

$$
\lim _{\left(t_{1}, t_{2}\right) \rightarrow(-\infty, 0)} \phi^{a}\left(t_{1}, t_{2}\right)=a(+\infty)
$$

Proof. Suppose that $a(-\infty)=0$. Let $\varepsilon>0$. Since $h_{j-1} \in L^{2}$ $(\mathbb{R})$, there exists $s_{0}>0$ such that

$$
\|a\|_{\infty} \int_{s_{0}}^{\infty}\left(h_{j-1}\left(s_{1}\right)\right)^{2} d s_{1}<\frac{\varepsilon}{2} .
$$

Take into account $\int_{-\infty}^{\infty}\left(h_{j-1}\left(s_{1}\right)\right)^{2} d s_{1}=1$ in the following computation

$$
\begin{aligned}
\left|\phi^{a}\left(t_{1}, t_{2}\right)\right|= & \left|\int_{-\infty}^{\infty} a\left(\frac{-t_{1}+s_{1}}{2 \sqrt{t_{2}\left(t_{1}^{2}+1\right)}}\right)\left(h_{j-1}\left(s_{1}\right)\right)^{2} d s_{1}\right| \\
\leq & \int_{-\infty}^{s_{0}}\left|a\left(\frac{-t_{1}+s_{1}}{2 \sqrt{t_{2}\left(t_{1}^{2}+1\right)}}\right)\left(h_{j-1}\left(s_{1}\right)\right)^{2}\right| d s_{1} \\
& +\int_{s_{0}}^{\infty}\left|a\left(\frac{-t_{1}+s_{1}}{2 \sqrt{t_{2}\left(t_{1}^{2}+1\right)}}\right)\left(h_{j-1}\left(s_{1}\right)\right)^{2}\right| d s_{1} \\
\leq & \max _{-\infty<s_{1}<s_{0}}\left|a\left(\frac{-t_{1}+s_{1}}{2 \sqrt{t_{2}\left(t_{1}^{2}+1\right)}}\right)\right|+\frac{\varepsilon}{2} .
\end{aligned}
$$

Since $a(s)$ converges to zero at $-\infty$, there exists $N_{1}>0$ such that $|a(s)|<\varepsilon / 2$ for $-s>N_{1}$. Take $\delta=1 /($ $\left.16 N_{1}^{2}\right)$. Then, we have $1 /\left(2 \sqrt{t_{2}}\right)>2 N_{1}$ for $0<t_{2}<\delta$. On the other hand, assume $t_{1}>s_{0}$ and $-\infty<s_{1}<s_{0}$. Then

$$
\frac{t_{1}-s_{1}}{\sqrt{t_{1}^{2}+1}}>\frac{t_{1}-s_{0}}{\sqrt{t_{1}^{2}+1}} .
$$


The right-hand side of this inequality converges to 1 when $t_{1}$ tends to $+\infty$; thus, there exists $N_{2}>s_{0}$ such that $\left(t_{1}-s_{0}\right) / \sqrt{t_{1}^{2}+1}>1 / 2$ for $t_{1}>N_{2}$. Consequently,

$$
N_{1}=2 N_{1} \frac{1}{2}<\frac{1}{2 \sqrt{t_{2}}} \frac{t_{1}-s_{0}}{\sqrt{\left(t_{1}^{2}+1\right)}}<\frac{t_{1}-s_{1}}{2 \sqrt{t_{2}\left(t_{1}^{2}+1\right)}} .
$$

For $0<t_{2}<\delta$ and $t_{1}>N:=\max \left\{s_{0}, N_{2}\right\}$, we have

$$
\left|a\left(\frac{-t_{1}+s_{1}}{2 \sqrt{t_{2}\left(t_{1}^{2}+1\right)}}\right)\right|<\frac{\varepsilon}{2} .
$$

We define $\tilde{a}(s)=a(s)-a_{2}$ in the case $a(-\infty) \neq 0$, where $a_{2}:=a(-\infty)$ is a constant. Note that $\tilde{a}(s)$ converges to zero at $-\infty$, and $\phi^{a_{1}+a_{2}}=\phi^{a_{1}}+\phi^{a_{2}}$ for any nilpotent symbols $a_{1}$ and $a_{2}$. Then

$$
\begin{aligned}
\lim _{\left(t_{1}, t_{2}\right) \rightarrow(+\infty, 0)} \phi^{a}\left(t_{1}, t_{2}\right)= & \lim _{\left(t_{1}, t_{2}\right) \rightarrow(+\infty, 0)} \phi^{\tilde{a}+a_{2}}\left(t_{1}, t_{2}\right) \\
= & \lim _{\left(t_{1}, t_{2}\right) \rightarrow(+\infty, 0)} \phi^{\tilde{a}}\left(t_{1}, t_{2}\right) \\
& +a_{2} \int_{-\infty}^{\infty}\left(h_{j-1}\left(s_{1}\right)\right)^{2} d s_{1}=a(-\infty) .
\end{aligned}
$$

Finally, the limit of $\phi^{a}\left(t_{1}, t_{2}\right)$ at $(-\infty, 0)$ can be proved analogously.

Lemma 8. Let $t_{0} \in \mathbb{R}_{+}$. If $a \in L^{\infty}(\mathbb{R})$ is continuous at $-1 /(2$ $\left.\sqrt{t_{0}}\right)$, then the spectral function $\phi^{a}$ satisfies

$$
\lim _{\left(t_{1}, t_{2}\right) \rightarrow\left(+\infty, t_{0}\right)} \phi^{a}\left(t_{1}, t_{2}\right)=a\left(-\frac{1}{2 \sqrt{t_{0}}}\right) .
$$

Analogously, if $a$ is continuous at $1 /\left(2 \sqrt{t_{0}}\right)$, then

$$
\lim _{\left(t_{1}, t_{2}\right) \rightarrow\left(-\infty, t_{0}\right)} \phi^{a}\left(t_{1}, t_{2}\right)=a\left(\frac{1}{2 \sqrt{t_{0}}}\right) .
$$

Proof. Suppose that $a$ converges to zero at $-1 /\left(2 \sqrt{t_{0}}\right)$. Let $\varepsilon>0$. Since $h_{j-1} \in L^{2}(\mathbb{R})$, there exists $s_{0}>0$ such that

$$
\|a\|_{\infty} \int_{-\infty}^{-s_{0}}\left(h_{j-1}\left(s_{1}\right)\right)^{2} d s_{1}<\frac{\varepsilon}{3}, \quad\|a\|_{\infty} \int_{s_{0}}^{\infty}\left(h_{j-1}\left(s_{1}\right)\right)^{2} d s_{1}<\frac{\varepsilon}{3} .
$$

Take into account $\int_{-\infty}^{\infty}\left(h_{j-1}\left(s_{1}\right)\right)^{2} d s_{1}=1$ in the following computation

$$
\begin{aligned}
\left|\phi^{a}\left(t_{1}, t_{2}\right)\right| \leq & \int_{-\infty}^{-s_{0}}\left|a\left(\frac{-t_{1}+s_{1}}{2 \sqrt{t_{2}\left(t_{1}^{2}+1\right)}}\right)\right|\left(h_{j-1}\left(s_{1}\right)\right)^{2} d s_{1} \\
& +\int_{-s_{0}}^{s_{0}}\left|a\left(\frac{-t_{1}+s_{1}}{2 \sqrt{t_{2}\left(t_{1}^{2}+1\right)}}\right)\right|\left(h_{j-1}\left(s_{1}\right)\right)^{2} d s_{1} \\
& +\int_{s_{0}}^{\infty}\left|a\left(\frac{-t_{1}+s_{1}}{2 \sqrt{t_{2}\left(t_{1}^{2}+1\right)}}\right)\right|\left(h_{j-1}\left(s_{1}\right)\right)^{2} d s_{1} \\
< & \frac{2 \varepsilon}{3}+\max _{-s_{0}<s_{1}<s_{0}}\left|a\left(\frac{-t_{1}+s_{1}}{2 \sqrt{t_{2}\left(t_{1}^{2}+1\right)}}\right)\right|
\end{aligned}
$$

Because of the continuity of $a(s)$ at $-1 /\left(2 \sqrt{t_{0}}\right)$, there exists $\delta_{1}>0$ such that $|a(s)|<\varepsilon / 3$ for $\mid s-(-1) /(2$ $\left.\sqrt{t_{0}}\right) \mid<\delta_{1}$. Let us estimate the value of the argument of $a$ :

$$
\begin{aligned}
I:= & \left|\frac{1}{2 \sqrt{t_{2}\left(t_{1}^{2}+1\right)}}\left(-t_{1}+s_{1}\right)-\frac{-1}{2 \sqrt{t_{0}}}\right| \\
\leq & \left|-\frac{1}{2 \sqrt{t_{2}}}+\frac{1}{2 \sqrt{t_{0}}}\right|\left|\frac{t_{1}}{\sqrt{t_{1}^{2}+1}}\right|+\frac{1}{2 \sqrt{t_{0}}}\left|1-\frac{t_{1}}{\sqrt{t_{1}^{2}+1}}\right| \\
& +\left|\frac{s_{1}}{2 \sqrt{t_{2}\left(t_{1}^{2}+1\right)}}\right| .
\end{aligned}
$$

Choose $\delta>0$ in such a way $\left|-\left(1 /\left(2 \sqrt{t_{2}}\right)\right)+\left(1 /\left(2 \sqrt{t_{0}}\right)\right)\right|$ $<\delta_{1} / 3$ for $\left|t_{2}-t_{0}\right|<\delta$. Pick $N_{1}>0$ such that $\mid 1-t_{1} /($ $\left.\sqrt{t_{1}^{2}+1}\right) \mid<\left(2 \sqrt{t_{0}} \delta_{1}\right) / 3$ whenever $t_{1}>N_{1}$. Now assume that $\mid$ $t_{2}-t_{0} \mid<\delta$ and $\left|s_{1}\right|<s_{0}$. Then, $\left|1 /\left(2 \sqrt{t_{2}}\right)\right|<1 /\left(2 \sqrt{t_{0}}\right)+\delta_{1} / 3$. Thus, $\left|s_{1}\right| /\left(2 \sqrt{t_{2}\left(t_{1}^{2}+1\right)}\right)$ converges to 0 when $t_{1}$ tends to + $\infty$. Therefore, there exists $N>N_{1}$ such that $\left|s_{1}\right| /(2$ $\left.\sqrt{t_{2}\left(t_{1}^{2}+1\right)}\right)<\delta_{1} / 3$ for $t_{1}>N$. The additional condition $t_{1}>$ $N$ implies

$$
\left|a\left(\frac{-t_{1}+s_{1}}{2 \sqrt{t_{2}\left(t_{1}^{2}+1\right)}}\right)\right|<\varepsilon / 3 .
$$

Hence, $\left|\phi^{a}\left(t_{1}, t_{2}\right)\right|<\varepsilon$ if $\left|t_{2}-t_{0}\right|<\delta$ and $t_{1}>N$.

If $a$ does not converge to zero at $-\left(1 /\left(2 \sqrt{t_{0}}\right)\right)$, then take the function $\tilde{a}(s)=a(s)-a_{2}$ and proceed as in the proof of Lemma 7 , where $a_{2}=a\left(-\left(1 /\left(2 \sqrt{t_{0}}\right)\right)\right)$.

Finally, the justification of the limit of $\phi^{a}\left(t_{1}, t_{2}\right)$ at $\left(-\infty, t_{0}\right)$ can be done analogously. 
Lemma 9. Let $a \in L^{\infty}(\mathbb{R})$ be continuous at $0 \in \mathbb{R}$. For $t_{0} \in \overline{\mathbb{R}}$, the spectral function $\phi^{a}$ satisfies

$$
\lim _{\left(t_{1}, t_{2}\right) \rightarrow\left(t_{0},+\infty\right)} \phi^{a}\left(t_{1}, t_{2}\right)=a(0) .
$$

Actually, we have uniform convergence of $\phi^{a}\left(t_{1}, t_{2}\right)$ at $\left(t_{0},+\infty\right)$, that is, for $\varepsilon>0$, there exists $N>0$ such that $\phi^{a}\left(t_{1}, t_{2}\right)-a(0) \mid<\varepsilon$ for all $t_{2}>N$ and for all $t_{1} \in \overline{\mathbb{R}}$.

Proof. Suppose that $a(0)=0$. Let $\varepsilon>0$, and choose $s_{0}>0$ such that equations (50) hold. Then

$$
\begin{aligned}
\left|\phi^{a}\left(t_{1}, t_{2}\right)\right| \leq & \int_{-\infty}^{-s_{0}}\left|a\left(\frac{-t_{1}+s_{1}}{2 \sqrt{t_{2}\left(t_{1}^{2}+1\right)}}\right)\right|\left(h_{j-1}\left(s_{1}\right)\right)^{2} d s_{1} \\
& +\int_{-s_{0}}^{s_{0}}\left|a\left(\frac{-t_{1}+s_{1}}{2 \sqrt{t_{2}\left(t_{1}^{2}+1\right)}}\right)\right|\left(h_{j-1}\left(s_{1}\right)\right)^{2} d s_{1} \\
& +\int_{s_{0}}^{\infty}\left|a\left(\frac{-t_{1}+s_{1}}{2 \sqrt{t_{2}\left(t_{1}^{2}+1\right)}}\right)\right|\left(h_{j-1}\left(s_{1}\right)\right)^{2} d s_{1} \\
< & \frac{2 \varepsilon}{3}+\max _{-s_{0}<s_{1}<s_{0}}\left|a\left(\frac{-t_{1}+s_{1}}{2 \sqrt{t_{2}\left(t_{1}^{2}+1\right)}}\right)\right|
\end{aligned}
$$

By the continuity of $a(s)$ at 0 , there exists $\delta_{1}>0$ such that $|a(s)|<\varepsilon / 3$ for $|s|<\delta_{1}$. For $-s_{0}<s_{1}<s_{0}$, we have

$\left|\frac{-t_{1}+s_{1}}{2 \sqrt{t_{2}\left(t_{1}^{2}+1\right)}}\right| \leq \frac{1}{2 \sqrt{t_{2}}}\left(\left|\frac{t_{1}}{\sqrt{t_{1}^{2}+1}}\right|+\frac{\left|s_{1}\right|}{\sqrt{t_{1}^{2}+1}}\right)<\frac{1}{2 \sqrt{t_{2}}}\left(1+s_{0}\right)$.

Take $N=\left(1+s_{0}\right)^{2} /\left(4 \delta_{1}^{2}\right)$. The inequality $t_{2}>N$ implies $1 /\left(2 \sqrt{t_{2}}\right)<\delta_{1} /\left(1+s_{0}\right)$. Thus, if $t_{2}>N, t_{1} \in \overline{\mathbb{R}}$, and $-s_{0}<s_{1}$ $<s_{0}$, then

$$
\left|\frac{-t_{1}+s_{1}}{2 \sqrt{t_{2}\left(t_{1}^{2}+1\right)}}\right|<\delta_{1} \text {. }
$$

Consequently, $\left|\phi^{a}\left(t_{1}, t_{2}\right)\right|<\varepsilon$ for all $t_{2}>N$ and $t_{1} \in \overline{\mathbb{R}}$.

Finally, in the case $a(0) \neq 0$, the proof can be carry out by considering the symbol $\tilde{a}(s)=a(s)-a(0)$.

For each nilpotent symbol $a\left(\operatorname{Im} \zeta_{1}\right) \in C(\overline{\mathbb{R}})$, the spectral function $\phi^{a}$ is continuous on $\bar{\Pi}$ and is constant along $\overline{\mathbb{R}} \times\{+\infty\}$. For this reason, the $C^{*}$-algebra generated by all spectral functions $\phi^{a}$ is not $C(\bar{\Pi})$, but it coin- cides with the algebra of continuous functions on a quotient space of $\bar{\Pi}$.

5.2. Toeplitz Operators $T_{a}$ with Continuous Symbols a $\left(\operatorname{Im} \zeta_{1}\right)$ . Introduce the quotient space $\Delta:=\bar{\Pi} /(\overline{\mathbb{R}} \times\{+\infty\})$. By Lemma 9, the spectral function $\phi^{a}$ can be identified with a continuous function on $\Delta$, which will be also denoted by $\phi^{a}: \Delta \longrightarrow \mathbb{C}$. We establish now one of our main results in this work.

Theorem 10. The $C^{*}$-algebra generated by all spectral functions $\phi^{a}$, with $a\left(\operatorname{Im} \zeta_{1}\right) \in C(\overline{\mathbb{R}})$, is isomorphic and isometric to the algebra $C(\Delta)$. That is, the $C^{*}$-algebra generated by all Toeplitz operators $T_{a}$ is isomorphic to $C(\Delta)$, where the isomorphism is defined on the generators by the rule

$$
T_{a} \mapsto \phi^{a} .
$$

Proof. The functions $\phi^{a}$ separate the points of $\Delta$ according to Lemmas 11-13 below. The Stone-Weierstrass theorem completes the proof.

Lemma 11. Let $\left(t_{1}, t_{2}\right)$ and $\left(y_{1}, y_{2}\right)$ be distinct points of $\bar{\Pi} \backslash \Pi$, where they do not belong simultaneously to $\overline{\mathbb{R}}$ $\times\{+\infty\}$. Then, there exists $a \in C(\overline{\mathbb{R}})$ such that $\phi^{a}\left(t_{1}\right.$, $\left.t_{2}\right) \neq \phi^{a}\left(y_{1}, y_{2}\right)$.

Proof. First consider the nilpotent symbol $a_{1}(s)=s /\left(\sqrt{s^{2}+1}\right)$, which is continuous on $\overline{\mathbb{R}}$. Note that

(i) $\phi^{a_{1}}\left( \pm \infty, t_{2}\right)=a_{1}\left(\mp 1 /\left(2 \sqrt{t_{2}}\right)\right)=\mp 1 / \sqrt{1+4 t_{2}}$ for $0<t_{2} \leq+\infty$,

(ii) $\phi^{a_{1}}( \pm \infty, 0)=a_{1}(\mp \infty)=\mp 1$,

(iii) $\phi^{a_{1}}\left(t_{1},+\infty\right)=a_{1}(0)=0$ for $t_{1} \in \overline{\mathbb{R}}$,

(iv) $\phi^{a_{1}}\left(t_{1}, 0\right)=-\int_{-\infty}^{t_{1}}\left(h_{j-1}\left(s_{1}\right)\right)^{2} d s_{1}+\int_{t_{1}}^{\infty}\left(h_{j-1}\left(s_{1}\right)\right)^{2} d s_{1}$ for $t_{1} \in \mathbb{R}$.

From $\int_{-\infty}^{\infty}\left(h_{j-1}\left(s_{1}\right)\right)^{2} d s_{1}=1$, we get

$$
\phi^{a_{1}}\left(t_{1}, 0\right)=2 \int_{t_{1}}^{\infty}\left(h_{j-1}\left(s_{1}\right)\right)^{2} d s_{1}-1
$$

This formula also says that $\phi^{a_{1}}( \pm \infty, 0)=\mp 1$. On the other hand, the Hermite function $h_{j-1}$ is continuous, and it has just a finitely many roots. Hence, $\phi^{a_{1}}\left(t_{1}, 0\right)$ is monotonically decreasing with respect to $t_{1}$. Thus, points in $\overline{\mathbb{R}} \times\{0\}$ are separated by $\phi^{a_{1}}$.

Recall that $\overline{\mathbb{R}} \times\{+\infty\}$ is identified with one point in $\Delta$ so take $(-\infty,+\infty)$ as representative point of the equivalence class $\overline{\mathbb{R}} \times\{+\infty\}$. For $t_{2}, \tilde{t}_{2} \in[0, \infty)$, the three points $\left(-\infty, t_{2}\right)$, $(-\infty,+\infty)$, and $\left(+\infty, \tilde{t}_{2}\right)$ are separated by $\phi^{a_{1}}( \pm \infty, t)$ because of the injective property of $1 / \sqrt{1+4 t}$.

Consider now the nilpotent symbol $a_{2}(s)=1 /\left(s^{2}+1\right)$, which is continuous on $\overline{\mathbb{R}}$. We have 
(i) $\phi^{a_{2}}\left( \pm \infty, t_{2}\right)=a_{2}\left(\mp 1 /\left(2 \sqrt{t_{2}}\right)\right)=4 t_{2} /\left(1+4 t_{2}\right)>0$ for $t_{2}>0$,

(ii) $\phi^{a_{2}}\left(t_{1},+\infty\right)=a_{2}(0)=1$ for $t_{1} \in \overline{\mathbb{R}}$,

(iii) $\phi^{a_{2}}\left(t_{1}, 0\right)=0$ for all $t_{1} \in \overline{\mathbb{R}}$.

Thus, $\phi^{a_{2}}$ separates each point $\left(t_{1}, 0\right)$ from the points $\left( \pm \infty, t_{2}\right)$ and $\left(t_{1},+\infty\right)$.

Now our aim is to separate the points of $\Pi$. Consider the following family of continuous functions:

$$
a_{\alpha}(s)=\left\{\begin{array}{lll}
0 & \text { si } & -\infty \leq s \leq-\alpha \\
\frac{1}{\alpha} s+1 & \text { si } & -\alpha \leq s \leq 0 \\
1 & \text { si } & 0 \leq s \leq+\infty
\end{array}\right.
$$

Then, $\phi^{a_{\alpha}}\left(t_{1}, t_{2}\right)=\psi^{\alpha}\left(t_{1}, t_{2}\right)+\varphi\left(t_{1}\right)$, where

$$
\psi^{\alpha}\left(t_{1}, t_{2}\right)=\int_{t_{1}-2 \alpha \sqrt{t_{2}\left(t_{1}^{2}+1\right)}}^{t_{1}}\left(\frac{s_{1}-t_{1}}{2 \alpha \sqrt{t_{2}\left(t_{1}^{2}+1\right)}}+1\right)\left(h_{j-1}\left(s_{1}\right)\right)^{2} d s_{1},
$$

$$
\varphi\left(t_{1}\right)=\int_{t_{1}}^{\infty}\left(h_{j-1}\left(s_{1}\right)\right)^{2} d s_{1} .
$$

Lemma 12. If $\left(t_{1}, t_{2}\right)$ and $\left(y_{1}, y_{2}\right)$ are distinct points in $\Pi$, then there exists $\alpha>0$ such that $\phi^{a_{\alpha}}\left(t_{1}, t_{2}\right) \neq \phi^{a_{\alpha}}\left(y_{1}, y_{2}\right)$, where $a_{\alpha}$ is defined in (60).

Proof. At first suppose that $\left(t_{1}, t_{2}\right)$ and $\left(y_{1}, y_{2}\right)$ satisfy $t_{1}<y_{1}$. Introduce $k:=\varphi\left(t_{1}\right)-\varphi\left(y_{1}\right)>0$. It is easy to see that $\psi^{\alpha}\left(t_{1}, t_{2}\right)$ can be written as

$\psi^{\alpha}\left(t_{1}, t_{2}\right)=\int_{-1}^{0}(s+1)\left[h_{j-1}\left(t_{1}+2 \alpha \sqrt{t_{2}\left(t_{1}^{2}+1\right)} s\right)\right]^{2} 2 \alpha \sqrt{t_{2}\left(t_{1}^{2}+1\right)} d s$. that

This integral representation allows us to prove easily

$$
\lim _{\alpha \rightarrow 0^{+}} \psi^{\alpha}\left(t_{1}, t_{2}\right)=0
$$

Take $\alpha$ small enough that $\left|\psi^{\alpha}\left(t_{1}, t_{2}\right)-\psi^{\alpha}\left(y_{1}, y_{2}\right)\right|<k$ 12. Then

$$
\begin{aligned}
\left|\phi^{a_{\alpha}}\left(t_{1}, t_{2}\right)-\phi^{a_{\alpha}}\left(y_{1}, y_{2}\right)\right| & =\left|k+\psi^{\alpha}\left(t_{1}, t_{2}\right)-\psi^{\alpha}\left(y_{1}, y_{2}\right)\right| \\
& \geq|k-| \psi^{\alpha}\left(t_{1}, t_{2}\right)-\psi^{\alpha}\left(y_{1}, y_{2}\right)|| \\
& \geq k-\frac{k}{2}>0 .
\end{aligned}
$$

Now suppose that $t_{1}=y_{1}=t$ and $t_{2}<y_{2}$. Let $\alpha=1 /$ $\left(2 \sqrt{t^{2}+1}\right)>0$. Then

$$
\begin{aligned}
& \psi^{\alpha}\left(t, t_{2}\right)=\int_{t-\sqrt{t_{2}}}^{t}\left(\frac{s_{1}-t}{\sqrt{t_{2}}}+1\right)\left(h_{j-1}\left(s_{1}\right)\right)^{2} d s_{1}, \\
& \psi^{\alpha}\left(t, y_{2}\right)=\int_{t-\sqrt{y_{2}}}^{t}\left(\frac{s_{1}-t}{\sqrt{y_{2}}}+1\right)\left(h_{j-1}\left(s_{1}\right)\right)^{2} d s_{1} .
\end{aligned}
$$

Since $0<t_{2}<y_{2}$, we have $t-\sqrt{y_{2}}<t-\sqrt{t_{2}}$. Besides, the following inequality holds

$$
0<\frac{s_{1}-t}{\sqrt{t_{2}}}+1<\frac{s_{1}-t}{\sqrt{y_{2}}}+1
$$

for $t-\sqrt{t_{2}}<s_{1}<t$. Hence, $\psi^{\alpha}\left(t, t_{2}\right)<\psi^{\alpha}\left(t, y_{2}\right)$. Finally, from $\varphi\left(t_{1}\right)=\varphi\left(y_{1}\right)=\varphi(t)$, we get $\phi^{a_{\alpha}}\left(t, t_{2}\right)<\phi^{a_{\alpha}}\left(t, y_{2}\right)$.

Lemma 13. Let $\left(t_{1}, t_{2}\right) \in \Pi$ and $\left(y_{1}, y_{2}\right) \in \bar{\Pi} \backslash \Pi$. Then, there exists $\alpha>0$ such that $\phi^{a_{\alpha}}\left(t_{1}, t_{2}\right) \neq \phi^{a_{\alpha}}\left(y_{1}, y_{2}\right)$, where $a_{\alpha}$ is given in (60).

Proof. Take $\left(t_{1}, t_{2}\right) \in \Pi,\left(y_{1}, 0\right) \in \mathbb{R} \times\{0\}$, and $\alpha>0$. It is easy to see that $\psi^{a_{\alpha}}\left(t_{1}, t_{2}\right)>0$. Then, we have $\phi^{a_{\alpha}}\left(y_{1}, 0\right)=\varphi\left(y_{1}\right)$ $\leq \varphi\left(t_{1}\right)<\phi^{a_{\alpha}}\left(t_{1}, t_{2}\right)$ for $t_{1} \leq y_{1}$. Now suppose that $y_{1}<t_{1}$. Let $k:=\varphi\left(y_{1}\right)-\varphi\left(t_{1}\right)>0$. The inequality $\psi^{\alpha}\left(t_{1}, t_{2}\right)<k$ holds for $\alpha>0$ small enough. For such $\alpha$,

$$
\phi^{a_{\alpha}}\left(t_{1}, t_{2}\right)=\psi^{\alpha}\left(t_{1}, t_{2}\right)+\varphi\left(t_{1}\right)<\varphi\left(y_{1}\right)=\phi^{a_{\alpha}}\left(y_{1}, 0\right) .
$$

This proves that all points of $\Pi$ can be separated from points of $\mathbb{R} \times\{0\}$. On the other hand,

$$
\begin{aligned}
0 & <\phi^{a_{\alpha}}\left(t_{1}, t_{2}\right)=\psi^{\alpha}\left(t_{1}, t_{2}\right)+\varphi\left(t_{1}\right) \\
& \leq \int_{t_{1}-2 \alpha \sqrt{t_{2}\left(t_{1}^{2}+1\right)}}^{t_{1}}\left(h_{j-1}\left(s_{1}\right)\right)^{2} d s_{1}+\varphi\left(t_{1}\right) \\
& =\int_{t_{1}-2 \alpha \sqrt{t_{2}\left(t_{1}^{2}+1\right)}}^{\infty}\left(h_{j-1}\left(s_{1}\right)\right)^{2} d s_{1}<1 .
\end{aligned}
$$

For $y_{2} \in \overline{\mathbb{R}}_{+}$, we have $\phi^{a_{\alpha}}\left(-\infty, y_{2}\right)=a_{\alpha}\left(1 /\left(2 \sqrt{y_{2}}\right)\right)=1$; hence, $\phi^{a_{\alpha}}\left(t_{1}, t_{2}\right) \neq \phi^{a_{\alpha}}\left(-\infty, y_{2}\right)$.

Finally, consider the function $a(s):=a_{\alpha}(s-\alpha)$. Then, $\phi^{a}$ $\left(+\infty, y_{2}\right)=a\left(-\left(1 /\left(2 \sqrt{y_{2}}\right)\right)\right)=0$, and consequently, $\phi^{a}\left(t_{1}, t_{2}\right)$ $\neq \phi^{a}\left(+\infty, y_{2}\right)$.

\section{Toeplitz Operators with Piecewise Continuous Symbols $a\left(\operatorname{Im} \zeta_{1}\right)$}

Take a finite subset $S=\left\{\beta_{0}, \cdots, \beta_{m}\right\} \subset \mathbb{R}$ and let $P C(\overline{\mathbb{R}}, S)$ be the set of functions continuous on $\overline{\mathbb{R}} \backslash S$ and having one-side limit values at each point of $S$. In this section, we study the $C^{*}$ -algebra generated by all Toeplitz operators $T_{a}$, where $a(\mathrm{Im}$ $\left.\zeta_{1}\right) \in P C(\overline{\mathbb{R}}, S)$. Obviously, we have to study the algebra generated by the spectral functions $\phi^{a}$. To begin with, take the 
indicator function $\chi_{+}=\chi_{[0,+\infty]}$. Then, the spectral function $\phi^{\chi_{+}}$is continuous on $\bar{\Pi} \backslash(\overline{\mathbb{R}} \times\{+\infty\})$ according to Lemmas 5,7 , and 8 . Actually,

$$
\phi^{\chi_{+}}\left(t_{1}, t_{2}\right)=\int_{-\infty}^{\infty} \chi_{[0,+\infty]}\left(\frac{-t_{1}+s_{1}}{2 \sqrt{t_{2}\left(t_{1}^{2}+1\right)}}\right)\left(h_{k-1}\left(s_{1}\right)\right)^{2} d s_{1}=\varphi\left(t_{1}\right),
$$

where $\varphi$ is defined in (61). Hence, $\phi^{\chi_{+}}$is continuous on $\bar{\Pi}$ because $\varphi$ is. Of course, we have now a spectral function which is not constant along $\overline{\mathbb{R}} \times\{+\infty\}$ anymore.

For any $a \in P C(\overline{\mathbb{R}},\{0\})$, we have

$$
a(s)=\tilde{a}(s)+\left[a\left(0_{+}\right)-a\left(0_{-}\right)\right] \chi_{+}(s),
$$

where $a\left(0_{-}\right)$and $a\left(0_{+}\right)$are the one-side limits of $a$ at 0 , and $\tilde{a}(s)=a(s)+\left[a\left(0_{-}\right)-a\left(0_{+}\right)\right] \chi_{+}(s)$. This function has a removable discontinuity at 0 ; thus, $\phi^{a}$ is continuous on $\bar{\Pi}$.

Theorem 14. The $C^{*}$-algebra generated by all Toeplitz operators $T_{a}$, with $a\left(\operatorname{Im} \zeta_{1}\right) \in P C(\overline{\mathbb{R}},\{0\})$, is isomorphic and isometric to $C(\bar{\Pi})$. The isomorphism is defined on the generators by the rule

$$
T_{a} \mapsto \phi^{a}
$$

Proof. If $t_{1} \neq y_{1}$, the function $\phi^{\chi_{+}}$separates any two points $\left(t_{1}, t_{2}\right)$ and $\left(y_{1}, y_{2}\right) \in \bar{\Pi}$. By Lemma 12 , two points in $\Pi$ can be separated by $\phi^{a_{\alpha}}$, where $a_{\alpha}$ is the nilpotent symbol given in (60). Further,

$$
\phi^{a_{\alpha}}\left(t_{1}, 0\right)=\varphi\left(t_{1}\right)<\phi^{a_{\alpha}}\left(t_{1}, t_{2}\right)<1=a_{\alpha}(0)=\phi^{a_{\alpha}}\left(t_{1},+\infty\right) .
$$

We continue our study by introducing another point of discontinuity. Take the indicator function $\chi_{\beta}:=$ $\chi_{[\beta / 2,+\infty]}(s)$, where $\beta>0$. We have

$$
\begin{aligned}
\phi^{\chi_{\beta}}\left(t_{1}, t_{2}\right) & =\int_{-\infty}^{\infty} \chi_{[\beta / 2,+\infty]}\left(\frac{-t_{1}+s_{1}}{2 \sqrt{t_{2}\left(t_{1}^{2}+1\right)}}\right)\left(h_{k-1}\left(s_{1}\right)\right)^{2} d s_{1} \\
& =\int_{t_{1}+\beta \sqrt{t_{2}\left(t_{1}^{2}+1\right)}}^{\infty}\left(h_{k-1}\left(s_{1}\right)\right)^{2} d s_{1} .
\end{aligned}
$$

According to Lemmas 5 and 7-9, the spectral function $\phi^{\chi_{\beta}}$ is continuous on $\bar{\Pi}$, except at the point $\left(-\infty, 1 / \beta^{2}\right)$. For $\lambda_{0} \in \mathbb{R}, \phi^{\chi_{\beta}}$ takes the constant value $\varphi\left(\lambda_{0}\right)$ along the curve $t_{1}+\beta \sqrt{t_{2}\left(t_{1}^{2}+1\right)}=\lambda_{0}$. From this equation, we get

$$
t_{2}=f\left(t_{1}\right)=\frac{\left(t_{1}-\lambda_{0}\right)^{2}}{\beta^{2}\left(t_{1}^{2}+1\right)}, t_{1} \leq \lambda_{0} .
$$

The horizontal line $t_{2}=1 / \beta^{2}$ is an asymptote of the graph of $t_{2}=f\left(t_{1}\right)$, and of course, $\phi^{\chi_{\beta}}\left(t_{1}, f\left(t_{1}\right)\right)=\varphi\left(\lambda_{0}\right)$ for $t_{1} \leq \lambda_{0}$. Thus, the level curves of $\phi^{\chi_{\beta}}$ converge to the point $\left(-\infty, 1 / \beta^{2}\right)$; our aim is to separate them at $\left(-\infty, 1 / \beta^{2}\right)$ through a mapping $Y_{\beta}$ in such a way that $\phi^{\chi_{\beta}} \circ Y_{\beta}^{-1}$ is continuous on $\bar{\Pi}$.

Lemma 15. Let $g: \overline{\mathbb{R}} \longrightarrow[-1 / 2,1 / 2]$ be any bijective, smooth, and increasing function, with $g(0)=0$. Take $\beta>0$, and let $Y_{\beta}$ be the function on $\Pi$ defined by the rule

$$
Y_{\beta}\left(t_{1}, t_{2}\right)=\left(t_{1}, t_{2}\left[1+g\left(\lambda\left(t_{1}, t_{2}\right)\right)\right]\right),
$$

where $\lambda\left(t_{1}, t_{2}\right)=t_{1}+\beta \sqrt{t_{2}\left(t_{1}^{2}+1\right)}$. Then, $Y_{\beta}$ is an homeomorphism from $\Pi$ onto itself, which can be continuously extended to $\bar{\Pi} \backslash\left\{\left(-\infty, 1 / \beta^{2}\right)\right\}$ with range $\bar{\Pi} \backslash\left(\{-\infty\} \times J_{\beta}\right)$, where $J_{\beta}=\left\{y: .5 / \beta^{2} \leq y \leq 1.5 / \beta^{2}\right\}$.

Proof. The function $h\left(t_{1}, t_{2}\right):=1+g\left(\lambda\left(t_{1}, t_{2}\right)\right)$ has range contained in $[1 / 2,3 / 2]$. Hence, $Y_{\beta}(\Pi) \subset \Pi$. Now suppose that $Y_{\beta}\left(t_{1}, t_{2}\right)=Y_{\beta}(a, b)$. Then, $t_{1}=a$ and $t_{2} h\left(a, t_{2}\right)=b h$ $(a, b)$. The function $h\left(a, t_{2}\right)$ is strictly increasing with respect to $t_{2}$. Thus, $t_{2}>b$ implies that $t_{2} h\left(a, t_{2}\right)>b h(a, b)$. Consequently, $Y_{\beta}$ is injective. Let $(c, d)$ be a point in $\Pi$. Consider the equation $Y_{\beta}\left(t_{1}, t_{2}\right)=(c, d)$, which is equivalent to the system of equations $t_{1}=c, t_{2} h\left(t_{1}, t_{2}\right)=d$. Thus, we have to prove that $t_{2} h\left(c, t_{2}\right)=d$ is solvable. The function $t_{2} h\left(c, t_{2}\right)$ is bijective from $(0,+\infty)$ into itself with respect to $t_{2}$. Therefore, $t_{2} h\left(c, t_{2}\right)=d$ has a unique solution. That is, $Y_{\beta}: \Pi$ $\longrightarrow \Pi$ is surjective. On the other hand, $Y_{\beta}$ is smooth and so is $Y_{\beta}^{-1}$ because of the Inverse Function Theorem.

The correspondence (76) also defines $Y_{\beta}$ on $\mathbb{R} \times\{0\}$ by $Y_{\beta}\left(t_{1}, 0\right)=\left(t_{1}, 0\right)$. Actually, $Y_{\beta}$ can be defined on $\bar{\Pi} \backslash$ $\left(\Pi \cup\left\{\left(-\infty, 1 / \beta^{2}\right)\right\}\right)$ according to the following limits:

$$
\begin{aligned}
\lim _{\left(t_{1}, t_{2}\right) \rightarrow\left(t_{1}^{0}, 0\right)} Y_{\beta}\left(t_{1}, t_{2}\right) & =\left(t_{1}^{0}, 0\right), t_{1}^{0} \in \overline{\mathbb{R}}, \\
\lim _{\left(t_{1}, t_{2}\right) \rightarrow\left(+\infty, t_{2}^{0}\right)} Y_{\beta}\left(t_{1}, t_{2}\right) & =\left(+\infty, \frac{3}{2} t_{2}^{0}\right), t_{2}^{0} \in \overline{\mathbb{R}}, \\
\lim _{\left(t_{1}, t_{2}\right) \rightarrow\left(t_{1}^{0},+\infty\right)} Y_{\beta}\left(t_{1}, t_{2}\right) & =\left(t_{1}^{0},+\infty\right), t_{1}^{0} \in \overline{\mathbb{R}}, \\
\lim _{\left(t_{1}, t_{2}\right) \rightarrow\left(-\infty, t_{2}^{0}\right)} Y_{\beta}\left(t_{1}, t_{2}\right) & =\left(-\infty, \frac{1}{2} t_{2}^{0}\right), 0<t_{2}^{0}<1 / \beta^{2}, \\
\lim _{\left(t_{1}, t_{2}\right) \rightarrow\left(-\infty, t_{2}^{0}\right)} Y_{\beta}\left(t_{1}, t_{2}\right) & =\left(-\infty, \frac{3}{2} t_{2}^{0}\right), t_{2}^{0}>1 / \beta^{2} .
\end{aligned}
$$

We will justify just the last limit. For $t_{1}<0$,

$$
\lambda\left(t_{1}, t_{2}\right)=\left|t_{1}\right|\left[-1+\beta \sqrt{t_{2}\left(1+\frac{1}{t_{1}^{2}}\right)}\right] .
$$


If $t_{2}^{0}>1 / \beta^{2}$ and $t_{2}$ is close enough to $t_{2}^{0}$, then $\lambda\left(t_{1}, t_{2}\right)$ tends to $+\infty$ when $t_{1}$ tends to $-\infty$. Thus, $\lim _{\left(t_{1}, t_{2}\right) \rightarrow\left(-\infty, t_{2}^{0}\right)} g$ $\left(t_{1}, t_{2}\right)=1 / 2$. Consequently,

$$
\lim _{\left(t_{1}, t_{2}\right) \rightarrow\left(-\infty, t_{2}^{0}\right)} t_{2} h\left(t_{1}, t_{2}\right)=\frac{3}{2} t_{2}^{0}
$$

that is, $Y_{\beta}\left(t_{1}, t_{2}\right)$ converges to $\left(-\infty, 3 t_{2}^{0} / 2\right)$ when $\left(t_{1}, t_{2}\right)$ tends to $\left(-\infty, t_{2}^{0}\right)$. A local analysis proves that $Y_{\beta}^{-1}$ is continuous. This completes the proof.

Lemma 16. The function $\phi^{\chi_{\beta}} \circ Y_{\beta}^{-1}$ is continuous on $\bar{\Pi}$, and it separates the points in the line segment $\{-\infty\} \times J_{\beta}$. For each continuous function $\phi: \bar{\Pi} \longrightarrow \mathbb{C}, \phi \circ Y_{\beta}^{-1}$ is also continuous on $\bar{\Pi}$ and has constant value along $\{-\infty\} \times J_{\beta}$.

With a discontinuity $\beta_{1}=\beta$, we define $\Phi_{1}=Y_{\beta}$. Introduce another point of discontinuity $\beta_{2}$, with $\beta_{1}<\beta_{2}$. Let $P_{2}$ $=\left(-\infty, 0.5 / \beta_{2}^{2}\right)$. The function $\phi^{\chi_{\beta_{2}} \circ \Phi_{1}^{-1}}$ has a continuous extension to $\bar{\Pi} \backslash\left\{P_{2}\right\}$, and its level curves $\Phi_{1}\left(t_{1},\left(t_{1}-\lambda\right)^{2} /(\right.$ $\left.\left.\beta_{2}^{2}\left(t_{1}^{2}+1,\right)\right)\right), t_{1}>\lambda$, converge to $P_{2}$. As in Lemma 15 , we can construct a mapping $\Phi_{2}$ that separates all these level curves, and $\phi^{\chi_{\beta_{2}} \circ \Phi_{1}^{-1} \circ \Phi_{2}^{-1}}$ is continuous on $\bar{\Pi}$. Adding more discontinuity points $\beta_{3}, \cdots, \beta_{m}$, with $\beta_{j}<\beta_{j+1}$, we can construct mappings $\Phi_{3}, \cdots, \Phi_{m}$ in such a way that $\phi^{\chi_{\beta_{m}}}$ 。 $\Phi_{1}^{-1} \circ \cdots \circ \Phi_{m}^{-1}$ is continuous on $\bar{\Pi}$.

Let $T_{S_{m}}$ denote the $C^{*}$-algebra generated by all Toeplitz operators $T_{a}$ with nilpotent symbols $a\left(\operatorname{Im} \zeta_{1}\right) \in P C\left(\overline{\mathbb{R}}, S_{m}\right)$, where $S_{m}=\left\{\beta_{0}, \cdots, \beta_{m}\right\}$. For simplicity in our explanation, we assume that $\beta_{0}=0$ and $S_{m-1} \subset S_{m} \subset \mathbb{R}_{+} \cup\{0\}$. We will explain how the Toeplitz algebra $T_{S_{m}}$ increases as $S_{m}$ does. By Theorem 14, $T_{S_{0}}$ is isomorphic to $C(\bar{\Pi})$, where the isomorphism is given on the generators by the rule

$$
\Psi_{0}: T_{S_{0}} \ni T_{a} \mapsto \phi^{a} \in C(\bar{\Pi})
$$

Consider now the algebra $T_{S_{1}}$, where $S_{1}=\left\{0, \beta_{1}\right\}$. By Lemma $16, \phi^{a} \circ \Phi_{1}^{-1}$ is continuous on $\bar{\Pi}$ for every $a \in P C(\overline{\mathbb{R}}$ , $\left.S_{1}\right)$. Then, the algebra $T_{S_{1}}$ is also isomorphic and isometric to $C(\bar{\Pi})$, where the isomorphism is given by

$$
\Psi_{1}: T_{S_{1}} \ni T_{a} \mapsto \phi^{a} \circ \Phi_{1}^{-1} \in C(\bar{\Pi}) .
$$

At first sight, both algebras $T_{S_{0}}$ and $T_{S_{1}}$ seem to have the same spectrum $\bar{\Pi}$, but they do not; they are identified with $C(\bar{\Pi})$ through different isomorphisms. Of course, $T_{S_{0}}$ is a subalgebra of $T_{S_{1}}$. If $T_{a} \in T_{S_{0}}$, then $\phi^{a} \circ \Phi_{1}^{-1} \in C(\bar{\Pi})$ and has constant value along the line segment $I_{1}=\{-\infty\} \times J_{\beta_{1}}$. According to the isomorphism $\Psi_{1}$, we can say that the spectrum of $T_{S_{1}}$ equals $\bar{\Pi}$; meanwhile, the spectrum of $T_{S_{1}}$ is the quotient space $\bar{\Pi} / I_{1}$. This phenomenon persists as long as the set $S_{m}$ grows.
Theorem 17. Let $S_{m}=\left\{0, \beta_{1}, \cdots, \beta_{m}\right\} \subset \mathbb{R}_{+}$. Then, there exist bijective continuous functions $\Phi_{j}: \Pi \longrightarrow \Pi, j=1, \cdots, m$, such that $\phi^{a} \circ \Phi_{1}^{-1} \circ \cdots \circ \Phi_{m}^{-1}$ admits a continuous extension to $\bar{\Pi}$ for each $a\left(\operatorname{Im} \zeta_{1}\right) \in P C\left(\overline{\mathbb{R}}, S_{m}\right)$. The $C^{*}$-algebra generated by all Toeplitz operators $T_{a}$ is isomorphic and isometric to $C(\bar{\Pi})$. The isomorphism is defined on the generators by the rule

$$
\Psi_{m}: T_{a} \mapsto \phi^{a} \circ \Phi_{1}^{-1} \circ \cdots \circ \Phi_{m}^{-1}
$$

Note that for each piecewise continuous symbol $a(\mathrm{Im}$ $\left.\zeta_{1}\right) \in P C\left(\overline{\mathbb{R}}, S_{m}\right)$, in general, the spectral function $\gamma^{a}: \Pi$ $\longrightarrow \mathbb{C}$ does not admit a continuous extension to $\bar{\Pi}$, but $\gamma^{a} \circ \Phi^{-1} \circ \Phi_{1}^{-1} \circ \cdots \circ \Phi_{m}^{-1}$ does, which means that $\gamma^{a}$ is uniformly continuous with respect to a new metric on $\Pi$; this metric is the pushforward of the usual metric using the mapping $\Phi^{-1} \circ \Phi_{1}^{-1} \circ \cdots \circ \Phi_{m}^{-1}$.

\section{Toeplitz Operators with \\ Symbols $a\left(\operatorname{Im} \zeta_{1}\right) b\left(\operatorname{Im} \zeta_{2}-\left|\zeta_{1}\right|^{2}\right)$}

In this section, we describe the $C^{*}$-algebra generated by all Toeplitz operators with symbols of the form $c\left(\zeta_{1}, \zeta_{2}\right)$ $=a\left(\operatorname{Im} \zeta_{1}\right) b\left(\operatorname{Im} \zeta_{2}-\left|\zeta_{1}\right|^{2}\right)$, where $a(s) \in C(\overline{\mathbb{R}})$, and $b(t) \epsilon$ $L^{\infty}\left(\mathbb{R}_{+}\right)$has limit values at $t=0,+\infty$. For such a symbol $c$, we have that $\gamma^{c}=\gamma^{a} \gamma^{b}$, which means that $T_{c}=T_{a} T_{b}$ $=T_{b} T_{a}$. Although $\gamma^{b}$ belongs to $C(\bar{\Pi})$, the spectral function

$$
\left(\gamma^{b} \circ \Phi^{-1}\right)\left(t_{1}, t_{2}\right)=\int_{\mathbb{R}_{+}} b\left(\frac{y_{2}}{2\left(t_{1}^{2}+1\right) t_{2}}\right)\left(\ell_{k-1}\left(y_{2}\right)\right)^{2} d y_{2}
$$

is continuous on $\bar{\Pi} \backslash\left\{P_{-}, P_{+}\right\}$, where $P_{-}=(-\infty, 0)$ and $P_{+}=(+\infty, 0)$. Since the level curves of $\gamma^{b}\left(x_{1}, x_{2}\right)$ are the horizontal lines $x_{2}=\mu$, the level curves of $\gamma^{b} \circ \Phi^{-1}$ are given by the equations $t_{2}=\mu /\left(t_{1}^{2}+1\right)$, with $\mu \in \mathbb{R}_{+}$.

Lemma 18. Let $f:[0,+\infty] \rightarrow[0,1]$ be any bijective, smooth, and increasing function. Then, the function

$$
\Theta\left(t_{1}, t_{2}\right)=\left(t_{1}, t_{2}+\frac{t_{1}^{2}}{t_{1}^{2}+1} f\left(t_{2}\left[t_{1}^{2}+1\right]\right)\right)
$$

is an homeomorphism from $\Pi$ onto itself, which can be continuously extended to $\bar{\Pi} \backslash\left\{P_{-}, P_{+}\right\}$with range $\bar{\Pi} \backslash I_{\infty}$, where $I_{\infty}=\left\{\left(\tau_{1}, \tau_{2}\right): \tau_{1}= \pm \infty\right.$ and $\left.0 \leq \tau_{2} \leq 1\right\}$. We have $\Theta\left( \pm \infty, t_{2}\right)$ $=\left( \pm \infty, t_{2}+1\right)$ for $0<t_{2}<+\infty$, and $\Theta$ acts like the identity mapping at the rest of points in $\bar{\Pi} \backslash\left(\Pi \cup\left\{P_{-}, P_{+}\right\}\right)$.

Proof. Similar to the proof of Lemma 15. 
The image of the level curve $t_{2}=\mu /\left(1+t_{1}^{2}\right)$ under $\Theta$ is the curve

$$
\tau_{2}=\frac{\mu}{\tau_{1}^{2}+1}+f(\mu) \frac{\tau_{1}^{2}}{\tau_{1}^{2}+1}
$$

This means that the level curves of $\gamma^{b} \circ \Phi^{-1} \circ \Theta^{-1}$ do not converge to a single point anymore.

Lemma 19. The function $\gamma^{b} \circ \Phi^{-1} \circ \Theta^{-1}$ is continuous on $\bar{\Pi}$, and for each continuous function $\phi: \bar{\Pi} \longrightarrow \mathbb{C}, \phi \circ \Theta^{-1}$ is also continuous on $\bar{\Pi}$ and has constant value along each component of $I_{\infty}$.

Theorem 20. The $C^{*}$-algebra generated by all Toeplitz operators $T_{a b}$, with $a\left(\operatorname{Im} \zeta_{1}\right) \in P C(\overline{\mathbb{R}},\{0\})$ and $b(t) \in L^{\infty}\left(\mathbb{R}_{+}\right)$ having limits values at $t=0,+\infty$, is isomorphic and isometric to $C(\bar{\Pi})$. The isomorphism is defined on the generators by the rule

$$
T_{a b} \mapsto \gamma^{a b} \circ \Phi^{-1} \circ \Theta^{-1} .
$$

For the Toeplitz operator $T_{\chi_{\beta}}$ with symbol $\chi_{\beta}=$ $\chi_{[\beta / 2,+\infty)}\left(\operatorname{Im} \zeta_{1}\right)$, there exists a mapping $\Theta_{\beta}: \Pi \longrightarrow \Pi$ such that $\gamma^{\chi_{\beta}} \circ \Phi^{-1} \circ \Theta^{-1} \circ \Theta_{\beta}^{-1}$ admits a continuous extension to $\bar{\Pi}$. The construction of $\Theta_{\beta}$ is similar to the construction of $\Phi_{\beta}$ given in Lemma 15, where one has to take into account the level curves of the spectral function $\gamma^{\chi_{\beta}} \circ \Phi^{-1} \circ \Theta^{-1}$, which converge to the point $\left(-\infty, 1+1 / \beta^{2}\right)$.

Implicitly, we have considered several compactifications of $\Pi$ associated to the $C^{*}$-algebras studied herein; each compactification depends on the kind of symbols. Take $Q_{-}=(-\infty,+\infty)$ and $Q_{+}=(+\infty,+\infty)$, let us explain the situation in the case of the algebra generated by the Toeplitz operators with symbols $a\left(\operatorname{Im} \zeta_{1}\right) \in C(\overline{\mathbb{R}})$. Essentially, the corresponding compactification of $\Pi$ is obtained from $\bar{\Pi} \backslash\left(\left\{Q_{-}, Q_{+}\right\}\right)$by gluing a line segment at each corner $Q_{-}$ and $Q_{+}$. Each spectral function $\gamma^{a}\left(x_{1}, x_{2}\right)$ is continuous on $\bar{\Pi} \backslash\left(\left\{Q-Q_{+}\right\}\right)$and has limit values when $\left(x_{1}, x_{2}\right)$ moves along the parabolas $x_{2}=\mu+\mu x_{1}^{2}$ and tends to $Q_{ \pm}$. For a net $\left\{X_{\lambda}\right\}$ tending to $Q_{+},\left\{\gamma^{a}\left(X_{\lambda}\right)\right\}$ converges if $\left\{X_{\lambda}\right\}$ is eventually in gaps between two parabolas close enough from each other with respect to the parameter $\mu$.

\section{Data Availability}

The data used to support the findings of this study are available from the corresponding author upon request.

\section{Conflicts of Interest}

The authors declare that they have no conflicts of interest.

\section{Acknowledgments}

This work was supported by Universidad Veracruzana under P/PROFEXCE-2020-30MSU0940B-22 project, México.

\section{References}

[1] R. Quiroga-Barranco and N. L. Vasilevski, "Commutative $C^{*}$ -Algebras of Toeplitz Operators on the Unit Ball, I. Bargmann-Type Transforms and Spectral Representations of Toeplitz Operators," Integral Equations and Operator Theory, vol. 59, no. 3, pp. 379-419, 2007.

[2] R. Quiroga-Barranco and N. L. Vasilevski, "Commutative $C^{*}$ -algebras of Toeplitz operators on the unit ball, II. Geometry of the level sets of symbols," Integral Equations and Operator Theory, vol. 60, no. 1, pp. 89-132, 2008.

[3] N. L. Vasilevski, Commutative Algebras of Toeplitz Operators on the Bergman Space. Operator Theory: Advances and Applications, vol. 185, Birkhäuser Verlag, Boston, 2008.

[4] C. Herrera-Yañez, E. A. Maximenko, and N. L. Vasilevski, "Vertical Toeplitz operators on the upper half-plane and very slowly oscillating functions," Integral Equations and Operator Theory, vol. 77, no. 2, pp. 149-166, 2013.

[5] C. Herrera-Yañez, O. Hutník, and E. A. Maximenko, "Les symboles verticaux, operateurs de Toeplitz sur les espaces ponderes de Bergman sur le demi-plan superieur et fonctions a oscillation tres lente," Comptes Rendus Mathematique, vol. 352, no. 2, pp. 129-132, 2014.

[6] W. Bauer, C. Herrera-Yañez, and N. L. Vasilevski, "Eigenvalue characterization of radial operators on weighted Bergman spaces over the unit ball," Integral Equations and Operator Theory, vol. 78, no. 2, pp. 271-300, 2014.

[7] K. Esmeral and E. A. Maximenko, "C $\mathrm{C}^{*}$-algebra of angular Toeplitz operators on Bergman spaces over the upper half-plane," Communications in Mathematical Analysis, vol. 17, no. 2, pp. 151-162, 2014.

[8] K. Esmeral, E. K. Maximenko, and N. L. Vasilevski, " $\mathrm{C}^{*}$-algebra generated by angular toeplitz operators on the weighted bergman spaces over the upper half-plane," Integral Equations and Operator Theory, vol. 83, no. 3, pp. 413-428, 2015.

[9] S. M. Grudsky, E. A. Maximenko, and N. L. Vasilevski, "Radial Toeplitz operators on the unit ball and slowly oscillating sequences," Communications in Mathematical Analysis, vol. 14, no. 2, pp. 77-94, 2013.

[10] J. Ramírez-Ortega and A. Sánchez-Nungaray, “Toeplitz operators with vertical symbols acting on the poly-Bergman spaces of the upper half-plane," Complex Analysis and Operator Theory, vol. 9, no. 8, pp. 1801-1817, 2015.

[11] N. L. Vasilevski, "On the structure of Bergman and polyBergman spaces," Integral Equations and Operator Theory, vol. 33, no. 4, pp. 471-488, 1999.

[12] M. Loaiza and J. Ramírez-Ortega, “Toeplitz operators with homogeneous symbols acting on the poly-Bergman spaces of the upper half-plane," Integral Equations and Operator Theory, vol. 87, no. 3, pp. 391-410, 2017.

[13] J. Ramírez-Ortega, M. Ramírez-Mora, and M. Morales-Ramos, "Algebra generated by a finite number of Toeplitz operators with homogeneous symbols acting on the poly-Bergman spaces," Operator Theory: Advances and Applications, vol. 279, pp. 383-402, 2020. 
[14] O. Hutník, "On Toeplitz-type operators related to wavelets," Integral Equations and Operator Theory, vol. 63, no. 1, pp. 29-46, 2009.

[15] O. Hutník, "Wavelets from Laguerre polynomials and Toeplitz-type operators," Integral Equations and Operator Theory, vol. 71, no. 3, pp. 357-388, 2011.

[16] J. Ramírez-Ortega, M. Ramírez-Mora, and A. Sánchez-Nungaray, "Toeplitz operators with vertical symbols acting on the poly-Bergman spaces of the upper half-plane II," Complex Analysis and Operator Theory, vol. 13, no. 5, pp. 2443-2462, 2019.

[17] K. Esmeral and E. A. Maximenko, "Radial Toeplitz operators on the Fock space and square-root-slowly oscillating sequences," Complex Analysis and Operator Theory, vol. 10, no. 7, pp. 1655-1677, 2016.

[18] K. Esmeral and N. L. Vasilevski, " $C^{*}$-algebra generated by horizontal Toeplitz operators on the Fock space," Boletín de la Sociedad Matemática Mexicana, vol. 22, no. 2, pp. 567-582, 2016.

[19] A. Sánchez-Nungaray, C. González-Flores, R. López-Martínez, and J. Arroyo-Neri, "Toeplitz operators with horizontal symbols acting on the poly-Fock spaces," Journal of Function Spaces, vol. 2018, Article ID 8031259, 8 pages, 2018.

[20] N. L. Vasilevski, "Poly-Fock spaces," in Differential Operators and Related Topics, vol. 117 of Operator Theory Advances and Applications, pp. 371-386, Springer, Basel AG, 2000.

[21] A. Sánchez-Nungaray and N. L. Vasilevski, "Toeplitz operators on the Bergman spaces with pseudodifferential defining symbols," Operator Theory: Advances and Applications, vol. 228, pp. 355-374, 2013.

[22] A. Sánchez-Nungaray and N. L. Vasilevski, "Algebras of Toeplitz operators on the three-dimentional Siegel domain," Integral Equations and Operator Theory, vol. 90, no. 4, pp. 1-42, 2018.

[23] J. Ramírez-Ortega and A. Sánchez-Nungaray, "Poly-Bergman type spaces on the Siegel domain," Communications in Mathematical Analysis, vol. 14, no. 2, pp. 113-128, 2013. 